\title{
1 Revealing importance of particles' surface functionalization on the properties of 2 magnetic alginate hydrogels
}

\section{3}

\author{
Mariusz Barczak ${ }^{1 *}$, Piotr Borowski ${ }^{1}$, Cristina Gila-Vilchez ${ }^{2,4}$, Miguel Alaminos ${ }^{3,4}$, Fernando González- \\ Caballero ${ }^{2,4}$, Modesto T. López-López ${ }^{2,4 *}$ \\ ${ }^{1}$ Department of Theoretical Chemistry, Institute of Chemical Sciences, Faculty of Chemistry, \\ Maria Curie-Skłodowska University, 20031 Lublin, Poland \\ ${ }^{2}$ Department of Applied Physics, Faculty of Sciences, University of Granada, 18071 Granada, Spain \\ ${ }^{3}$ Tissue Engineering Group, Department of Histology, University of Granada, Granada, Spain \\ ${ }^{4}$ Instituto de Investigación Biosanitaria ibs.GRANADA, Granada, Spain \\ Corresponding authors: M.B.: mbarczak@umcs.pl, M.T. L.-L.: modesto@ugr.es
}

Abstract:

Iron/silica core-shell microparticles (IMPs) were functionalized by different functional groups including amine, glycidoxy, phenyl, and thiocyanate. Many of the IMPs modifications are reported for the first time. The resulting surface chemistry turned out to affect the properties of magnetic alginate hydrogels fabricated from sodium alginate and dispersed IMPs. Differences in magnetorheological properties of the obtained magnetic hydrogels can be at least partially attributed to the interactions between alginate and surface functionalities of IMPs. Density Functional Theory (DFT) calculations were carried out to get detailed insight into those interactions in order to link them with the observed macroscopic properties of the obtained hydrogels. For example, amine groups on the IMPs surface resulted in well-formed hydrogels while the presence of thiocyanate or phenyl groups - in poorly formed ones. This observation can be used for tuning the properties of various carbohydrate-based hydrogels.

\section{Introduction:}

Hydrogels can be considered as three-dimensional, hydrophilic networks of flexible polymer chains swollen by water or other fluid. They are able to store a large amount of water (even up to thousands times their dry weight) while maintaining the structure that can be cast into practically any shape or form (Seliktar, 2012). They are soft and capable of retaining large amounts of water thus closely resemble living tissues. Mainly for that reason hydrogels are considered as particularly promising materials in the rapidly developing field of tissue engineering as matrices for replacing and regenerating 
different tissues and organs (Drury \& Mooney, 2003; Geckil, Xu, Zhang, Moon, \& Demirci, 2010; Peppas, Hilt, Khademhosseini, \& Langer, 2006). Besides they also continuously find widespread applications in the biomedical field including biosensors (Buenger, Topuz, \& Groll, 2012; L. Li et al., 2015; Ulijn et al., 2007), drug delivery systems (Fan, Tian, \& Liu, 2019; J. Li \& Mooney, 2016; Qiu \& Park) or wound healing materials (Dimatteo, Darling, \& Segura, 2018; Griffin, Weaver, Scumpia, Di Carlo, \& Segura, 2015; Liu \& Guo, 2018).

There is a plethora of different hydrogelators which can be used to fabricate hydrogels. Depending on hydrogelator origin, the resulting hydrogels can be: (i) natural polymer-based hydrogels, (ii) synthetic polymer-based hydrogels and (iii) supramolecular hydrogels (Du, Zhou, Shi, \& Xu, 2015). Natural polymer-based hydrogels are particularly useful in tissue engineering related applications due to their remarkable in vitro and in vivo biocompatibility, confirmed in many studies (De Groot et al., 2001; Kulkarni, Boppana, Krishna Mohan, Mutalik, \& Kalyane, 2012; Lai, 2010; C. Lee et al., 2013). Indeed, in the literature there is a continuously increasing number of papers reporting such potential biomedical applications with the use of alginate, chitosan, fibrin or collagen as the most prominent examples of natural polymer-based hydrogels; many recent reviews nicely summarize the current state-of-the-art (Dimatteo et al., 2018; X. Li, Sun, Li, Kawazoe, \& Chen, 2018; Mahinroosta, Jomeh Farsangi, Allahverdi, \& Shakoori, 2018; Mantha et al., 2019; Qureshi et al., 2019; Tu et al., 2019).

Among different hydrogels' types, alginate-based hydrogels are considered as one of the preferred formulations, mainly due to low cost and excellent biocompatibility of alginate hydrogelators (EsponaNoguera et al., 2018; Soon-Shiong et al., 1994; Wang et al., 2019). Alginates (i.e., sodium, potassium, calcium or magnesium salts of alginic acid) are biopolymers usually extracted from different species of seaweeds (macroalgae) such as, for example, Rhodophyceae (red macroalgae), Phaeophyceae and Laminaria (brown macroalgae) or Chlorophyceae (green macroalgae). From chemical point of view alginic acid is composed of unbranched chains of $\alpha$-L-guluronic acid (G-block) and $\beta$ - $D$-mannuronic acid (M-block) covalently linked by 1-4 glycosidic bond (Fig. S1). Alginates extracted from different species usually show variations in their chemical structure due to different sequences of G- and M-blocks (K. Y. Lee \& Mooney, 2012). In the presence of multivalent cations (e.g., calcium) alginates form a physical ionotropic hydrogel as a result of ionic crosslinking between the negatively charged polyionic alginate chains and multivalent cations. Negative charge of the alginate chains results from dissociation of $-\mathrm{COOH}$ (alginic acid) or - $\mathrm{COONa}$ (alginate) groups into carboxylate anions - $\mathrm{COO}^{-}$.

To fabricate "smart" materials, alginate hydrogels can be doped with magnetic particles. Incorporation of magnetically-susceptible species into hydrogel structure may provide additional 
features like stimuli-responsive action, sufficient biocompatibility or tailorable rheological properties (Gila-Vilchez, Duran, Gonzalez-Caballero, Zubarev, \& Lopez-Lopez, 2019; Konwar, Gogoi, \& Chowdhury, 2015; Supramaniam, Adnan, Mohd Kaus, \& Bushra, 2018). For those reasons, magnetic hydrogels are becoming even more useful for biomedical applications, particularly as scaffolds for soft tissue engineering, where the above-mentioned advantages are of paramount importance. The rheological properties of the magnetic hydrogels (also called ferrogels) in the presence of magnetic field are then predominantly controlled by the factors related to the type, size, shape and concentration of the incorporated magnetic particles (Bonhome-Espinosa et al., 2017; Gila-Vilchez et al., 2018; Gila-Vilchez, Duran, et al., 2019; Gila-Vilchez, Mañas-Torres, et al., 2019). For example, small particles (e.g., nanoparticles) experience a weak attraction between themselves under moderate magnetic field in contrast to bigger particles (e.g., microparticles) which are able to interact strongly even at low magnetic fields. Thus strong magnetic fields can provoke significant viscoelasticity changes of magnetic hydrogels composed of microparticles (Gila-Vilchez et al., 2018). The fascinating research area of magnetic hydrogels is, however, still at its infancy. There is a considerable number of reports about ferrogels but they do not fully reflect the high potential they have with regard to the current and emerging biomedical challenges. polymer filaments that form the hydrogels having a direct impact on the properties of the hydrogels, as has been recently shown (Bonhome-Espinosa et al., 2017). Furthermore, magnetic particles with the appropriate surface chemistry can conjugate drugs, proteins, enzymes or antibodies, which is required for numerous applications. For instance, it has been recently shown that medical treatment with magnetic particles conjugated by nerve growth factor significantly promotes neurite outgrowth and increases the complexity of the neuronal branching trees (Marcus, Skaat, Alon, Margel, \& Shefi, 2015).

As can be seen from the comprehensive set of representative literature presented above the magnetic particles are usually used as received, i.e., without any functionalization. Incorporation of nano- or micro-sized particles into hydrogel is based on the physical incorporation within the hydrogel, having the possible consequence of continuous release of the particles from the hydrogel matrix to the environment (Barbucci, Giani, Fedi, Bottari, \& Casolaro, 2012). However, apart from bulk iron providing magnetic field actuation, the surface of magnetic particles can be used to tune specific or nonspecific interactions with the hydrogelator moieties (Tanasa et al., 2019), which in turn can affect the final properties of resulting hydrogels and even provide more favorable features like better adhesion of biological species (e.g., cells). Functionalization with amine group with the use of 3- 
aminopropyltriethoxysilane or 3-aminopropyltrimethoxysilane is frequently reported strategy to modify

97 the surface of the particles and properties of resulting hydrogels (Barbucci et al., 2012; Čampelj, Makovec, \& Drofenik, 2009; Giani, Fedi, \& Barbucci, 2012; Long et al., 2015; Park et al., 2009; Zhu, Zheng, Wang, \& Wang, 2016). Unfortunately, in the literature there is few attempts to chemically functionalize magnetic particles using groups other than amines (Tanasa et al., 2019).

In this work we seek to determine the role of surface functionalization of iron particles on the properties of the resulting alginate magnetic hydrogels. We hypothesize that different surface chemistries of iron particles can affect chemical interactions between the both phases in a distinct way, and these changes will contribute to the different microstructure, mechanical properties, and biocompatibility of ferrogels. Alginate was chosen as a model matrix due to its high biocompatibility allowing its use in biomedical applications. A set of different surface functionalizations of iron/silica coreshell microparticles (IMPs) has been chosen. Interactions between specific surface groups and alginate chains have been elucidated with the aid of DFT quantum chemistry calculations to get a more detailed insight into those interactions.

\section{Experimental}

\subsection{Reagents}

Sodium alginate (ALG, MW: 20-40 kDa, Sigma Aldrich) was used as received. The relative content of mannuronic to guluronic acid was experimentally estimated using protocols based on ellipsometry (Donati et al., 2003; Morris, Rees, \& Thom, 1980) and FTIR spectroscopy (Filippov \& Kohn, 1974). The ellipsometric method showed that the composition of ALG is as follows: $75 \%$ guluronic acid, $17 \%$ mannuronic acid, and 8\% mixed sequences (cf. Fig. S1b); while FTIR method showed that it is: $82 \%$ guluronic acid and 18\% mannuronic acid (cf. Fig. S1c). Powder of sodium alginate, as received, had a bulk density of $0.509 \pm 0.017 \mathrm{~g} / \mathrm{mL}$ and the reported skeleton density of sodium alginate is $1.6010 \pm 0.0002$ $\mathrm{g} / \mathrm{mL}$ (Censi, Gigliobianco, Malaj, \& Di Martino, 2016). From this data, we estimated a porosity of the sodium alginate powder of $68.2 \pm 1.1 \%$ - for details on the bulk and skeletal density of aerogels see (Fitzpatrick, Staiger, Deb-Choudhury, \& Ranford, 2018). Calcium carbonate ( $\mathrm{CaCO}_{3}$, Sigma Aldrich), Dglucono- $\delta$-lactone (GDL, Sigma Aldrich), aminopropyltriethoxysilane (APTES, 97\%, Sigma-Aldrich), (3trimethoxysilylpropyl)diethylenetriamine (TMPMT, 95\%, Fluorochem), Nphenylaminomethyltriethoxysilane (PATES, 95\%, Fluorochem), thiocyanatopropyltriethoxysilane (TCTES, 95\%, Fluorochem), glycidoxypropyltrimethoxysilane (GPTMS, 98\%, Sigma Aldrich), phenyltriethoxysilane (PTES, 97\%, Gelest), sodium hydroxide ( $\mathrm{NaOH}$, Sigal), hydrochloric acid $(\mathrm{HCl}$, Sigal) were used without 
further purification. As magnetic particles we used silica-covered iron particles (CIP grade) supplied by BASF, Germany, referred in the test as IMPs.

\subsection{Functionalization of iron/silica core-shell microparticles}

$1312 \mathrm{~g}$ of IMPs were placed in a vial and mixed with $40 \mathrm{~mL}$ of absolute ethanol, previously acidified with 0.57 $132 \mathrm{~mL}$ of $1.75 \mathrm{M} \mathrm{HCl}$. The suspension was sonicated for $5 \mathrm{~min}$. After that time $1 \mathrm{mmol}$ of proper 133 organofunctional alkoxysilane (APTES, TMPMT, PTES, GPTMS, PATES or TCTES) was added and the suspension was submitted six times to the following sequence: $5 \mathrm{~min}$ of sonification and $25 \mathrm{~min}$ of stirring (in total 3 hours). After that time the IMPs were separated from the solution by magnet, washed two times with absolute ethanol and dried overnight at $40{ }^{\circ} \mathrm{C}$. The numbering of the samples together with the organofunctional alkoxysilane used to their synthesis is given in Table 1.

\subsection{Preparation of magnetic hydrogels}

For the preparation of the magnetic hydrogels we followed a two-step protocol proposed in a previous work (Gila-Vilchez et al., 2018), which allows the generation of magnetic hydrogels with excellent homogeneity and reproducibility. Briefly, ALG was dissolved in distilled water to prepare $1 \% \mathrm{w} / \mathrm{v}$ solution. Then, $9 \mathrm{mg}$ of $\mathrm{CaCO}_{3}$ was added to $6 \mathrm{~mL}$ of this solution, and the vial was vortexed for $1 \mathrm{~min}$. After that $32 \mathrm{mg}$ of GDL was added and the mixture was again vortexed for $1 \mathrm{~min}$. Then the solution was left in a closed vial for $90 \mathrm{~min}$ at room temperature. After that time the forming gelling mixture was

145 transferred to another vial, vortexed $(1 \mathrm{~min})$ and $0.7 \mathrm{~mL}$ was transferred do the Eppendorf vial containing specific amount of IMPs to reach the required final concentration of $0.9 \% \mathrm{v} / \mathrm{v}$ (note that from the initial amount of ALG mixture of $6 \mathrm{~mL}$ it is possible to prepare more IMP-ALG hydrogel samples of 0.7 $m L)$. The mixture of gel and IMPs in the Eppendorf vial was submitted to the following treatment steps to disperse well the IMPs within the gel: (i) vortex - $1 \mathrm{~min}$, (ii) sonification - $5 \mathrm{~min}$, (iii) vortex - $1 \mathrm{~min}$. After

151 atmosphere. The next day the formed hydrogels were submitted to the further analyses. In the case of 152 the non-magnetic reference hydrogel the preparation scheme was the same apart from the fact that 0.7 $\mathrm{mL}$ of the solution was transferred to the empty Eppendorf vial without IMPs.

\section{2.4. Physicochemical characterization of the IMPs}

155 IMPs were analyzed by several instrumental techniques. The nitrogen sorption measurements were performed at $-196{ }^{\circ} \mathrm{C}$ using a 1200 e sorption analyzer (Quantachrome). All samples were degassed at $157110^{\circ} \mathrm{C}$ in vacuum prior to measurements. The BET specific surface areas $\left(\mathrm{S}_{\mathrm{BET}}\right)$ were evaluated in the range of relative pressures of $0.05-0.20$. The total pore volumes $\left(V_{p}\right)$ were calculated by converting the 
amount of adsorbed nitrogen at relative pressure $\sim 0.99$ to the volume of liquid adsorbate. The SEM

160 imaging of randomly selected parts of the surface was performed under high vacuum conditions by 161 means of Quanta 3DFEG (FEI, USA) microscope with the accelerating voltage 5/20 kV. X-ray 162 photoelectron spectroscopy (XPS) spectra were obtained in high vacuum $\left(8 \times 10^{-9} \mathrm{~Pa}\right)$ by means of Multi163 Chamber Analytical System (Prevac, Poland) equipped with monochromatic $450 \mathrm{~W}$ Al K-alpha X-ray 164 radiation source. The binding energy scale was referenced against C $1 \mathrm{~s}=284.7 \mathrm{eV}$ line. Deconvolutions of 165 the obtained spectra were done using MultiPak software. To determine the surface pH $0.1 \mathrm{~g}$ of IMPs was 166 suspended in $50 \mathrm{~mL}$ of water and stabilized overnight before the measurement. The $\mathrm{pH}$ of the solution was then measured using a $510 \mathrm{pH}$-meter (Oakton Instruments). The zeta potential was evaluated using Zetasizer Nano ZS (Malvern Instruments). Suspensions were prepared by dispersing $~ 5 \mathrm{mg}$ of IMPs in $2 \mathrm{~mL}$ of $1 \times 10^{-3} \mathrm{M} \mathrm{KCl}$.

\subsection{Physicochemical characterization of the IMPs-ALG hydrogels}

171 The water-releasing tests were carried out for each hydrogel (in triplicate). Fresh hydrogel with known 172 mass ( $\sim .47-0.51 \mathrm{~g}$ ) was placed in a plastic vial and submitted to drying at room temperature. Weight 173 losses were recorded during drying at specific times during 14 hours (i.e., until the bottom of the vial 174 only dry residue remained and the weight did not change over time). Humidity was not controlled during 175 the experiments but it was the same for all the samples. The microscopic structure of the selected 176 hydrogels was analyzed by Scanning Electron Microscopy (SEM), accomplished using a FEI Quanta 400 177 ESEM equipped with a Peltier effect cooling stage. Before SEM analysis, the hydrogels were prepared 178 according to a well-established protocol (detailed information is provided in Supplementary data) and 179 subjected to $\mathrm{CO}_{2}$ critical point drying (Anderson, 1951). Differential scanning calorimetric (DSC) 180 measurements were carried out using a DSC 204 Netzsch calorimeter. The dynamic mode scans were 181 collected at a heating rate of $20^{\circ} \mathrm{C} \cdot \mathrm{min}^{-1}$, from $20{ }^{\circ} \mathrm{C}$ to $200{ }^{\circ} \mathrm{C}$ under argon flow $\left(20 \mathrm{~cm}^{3} \cdot \mathrm{min}^{-1}\right)$. 182 Aluminum pots were punched with a needle before each experiment.

\subsection{Rheological measurements of the IMPs-ALG hydrogels}

184 Rheological properties of the hydrogels were determined at room temperature using the MCR 300 185 magneto rheometer (Physica Anton Paar) using a plate-plate geometry of $20 \mathrm{~mm}$ of diameter. Linear 186 viscoelastic region (LVR) of the studied hydrogels was determined by subjecting them to deformation amplitude sweep tests at a constant frequency of $1 \mathrm{~Hz}$ and stepwise increasing shear strain amplitude, $Y_{0}$. From these measurements the values of the storage $\left(G^{\prime}\right)$ and loss $\left(G^{\prime \prime}\right)$ moduli as a function of $Y_{0}$ were determined along with the averaged $G^{\prime}$ and $G^{\prime \prime}$ values within the LVR region. Frequency sweep tests 
were done at a fixed shear strain amplitude $\left(\gamma_{0}=0.03 \%\right)$ within the LVR, and increasing frequency in the range from 0.15 to $15 \mathrm{~Hz}$. From these measurements the values of $G^{\prime}$ and $G^{\prime \prime}$ were determined as functions of frequency. Both amplitude and frequency scans were carried without and with the presence of magnetic field of two arbitrarily chosen intensities of $141 \mathrm{kA} \mathrm{m}^{-1}$ and $242 \mathrm{kA} \mathrm{m}^{-1}$.

\subsection{Cell viability assessment}

First, the viability of the human fibroblasts was analyzed using functional WST-1 assays (Cell Proliferation Reagent WST-1, Roche Diagnostics, Germany) based on the colorimetric transformation of tetrazolium salt (WST-1) to formazan driven by the activity of the mitochondrial dehydrogenase of living cells, which is directly proportional to the number of viable (i.e., metabolically active) cells. Fibroblasts were cultured for $48 \mathrm{~h}$ in contact with hydrogel, and the absorbance of the colorimetric reaction was inspected with an Assay UVM 340 spectrophotometer in triplicate.

To determine the structural integrity of cells cultured in contact with the biomaterials, the total DNA released by the cells corresponding to each condition was quantified with a NanoDrop 2000 UV-Vis Spectrophotometer (Thermo Fisher Scientific). $10 \mu \mathrm{L}$ of the culture medium was used to determine the amount of DNA for each condition and time. The release of DNA from cells into the culture medium occurs as a result of irreversible damage to the cell membrane. Therefore higher DNA concentration in the culture medium indicates a higher number of dead cells with the membrane structurally disrupted.

Finally, Live/Dead cell viability assays were used on cell cultured with the different types of materials evaluated in the present work. This method combines a functional assay based on calcein AM, which is metabolically activated and turns to green by living cells, and a structural assay based on ethidium homodimer-1, which can only enter to the cell nucleus if the cell is dead. Therefore, living cells are labeled in green and dead cells are labeled in red. Cells were cultured in the presence of each material for $48 \mathrm{~h}$ and washed in PBS. Then, calcein AM and ethidium homodimer-1 were added as suggested by the manufacturer, and representative micrographs were taken from human fibroblasts cultured for $48 \mathrm{~h}$ in contact with magnetic hydrogels using an A1R Nikon fluorescence microscope (Nikon) with constant illumination and capture parameters. Micrographs were analyzed using the NISElements and ImageJ v1.46 software packages, and the percentage of live and dead cells was calculated for each experimental condition.

\subsection{Theoretical calculations}

The Density Functional Theory (DFT) calculations were carried out at the DFT/B3LYP/6-311++G** 
221 were found first. The type of stationary point was determined by analysis of the obtained frequencies.

222 All calculated frequencies were real indicating that minima on PES were found. Calculations were 223 performed using the PQS quantum chemistry package (Baker et al., 2009). The relative energies, which 224 include zero-point vibrational energy (ZPVE) corrections, were calculated as $\Delta \mathrm{E}=\mathrm{E}$ (products)$225 E$ (substrates), thus the negative value of $\Delta E$ means that the products are more stable than substrates. 226 Following our previous findings (Barczak \& Borowski, 2019; Barczak, Wierzbicka, \& Borowski, 2018) we 227 considered interactions of alginate representative fragments and functionalities as the energetics is not 228 affected by the presence of the matrix.

\section{2.8. Statistical analysis}

230 Parametric analysis of variance (ANOVA) was used to determine differences between hydrogels with 231 respect to their water releasing profiles and storage modulus ( $\left.G^{\prime}\right)$. After ANOVA analysis, pairwise 232 multiple comparisons analysis was performed using Tukey HSD and Dunnett two-tailed post hoc tests. 233 For Dunnett test, the $\mathrm{R}$ hydrogel was a single control. A difference was considered to be statistically 234 significant if $p$ value was lower than 0.05 . Hydrogels biocompatibility (i.e. WST-1 and DNA quantification) 235 due to the lack of samples normality (as confirmed by Shapiro-Wilk test), was assessed using non236 parametric Kruskal-Wallis test, followed by multiple pairwise comparisons based on the Conover-Iman 237 post host test. A difference was considered statistically significant if the p-value was less than 0.05 238 corrected by Bonferroni correction.

\section{3. Results and discussion}

\section{3.1. Functionalization and characterization of iron microparticles}

242 The commercial iron core/shell particles (IMPs) used in this study are composed of spherical and 243 often aggregated units with a wide distribution of sizes. The SEM images of the initial particles (referred 244 as R) at different magnifications are presented in Fig. 1. The average size is around 2-3 $\mu \mathrm{m}$ and the 245 density of $7.71 \pm 0.19 \mathrm{~g} \mathrm{~cm}^{-3}$. The IMPs used in this study exhibit a typical ferromagnetic behavior with 246 saturation magnetization of approx. $1600 \mathrm{kA} \mathrm{m}^{-1}$ (Gila-Vilchez et al., 2018). The particles are covered, 247 though not perfectly, with a layer of silica thus there are many surface silanol groups present, which 248 makes the resulting surface very versatile and ready to be modified by attaching specific functional 249 groups. Due to that the grafting of different organosilica monomers was chosen in this study as the most 250 straightforward functionalization strategy. To accomplish it, six different monomers were tested - the 251 range of the monomers used is listed in Table 1; grafted functional groups are also presented in Fig. 1 252 and S2a. The reason for choosing such a wide range of functional groups was twofold: (i) examining 
253 whether functionalization of iron surface can be achieved using monomers other than APTES and (ii) 254 investigating the possible effect of chemistry of IMP surface layer on the properties of obtained alginate 255 magnetic hydrogels. The choice of functional groups used in this study was dictated by the fact that they 256 can interact with the alginate network in various ways. For example, amino or thiocyanate groups can 257 form hydrogen bonds with alginate oxygens, while phenyl or glycidoxy groups are expected to 258 repulsively interact with a negatively charged alginate hydrogel network. 
Table 1. Structural and chemical properties of the functionalized iron particles

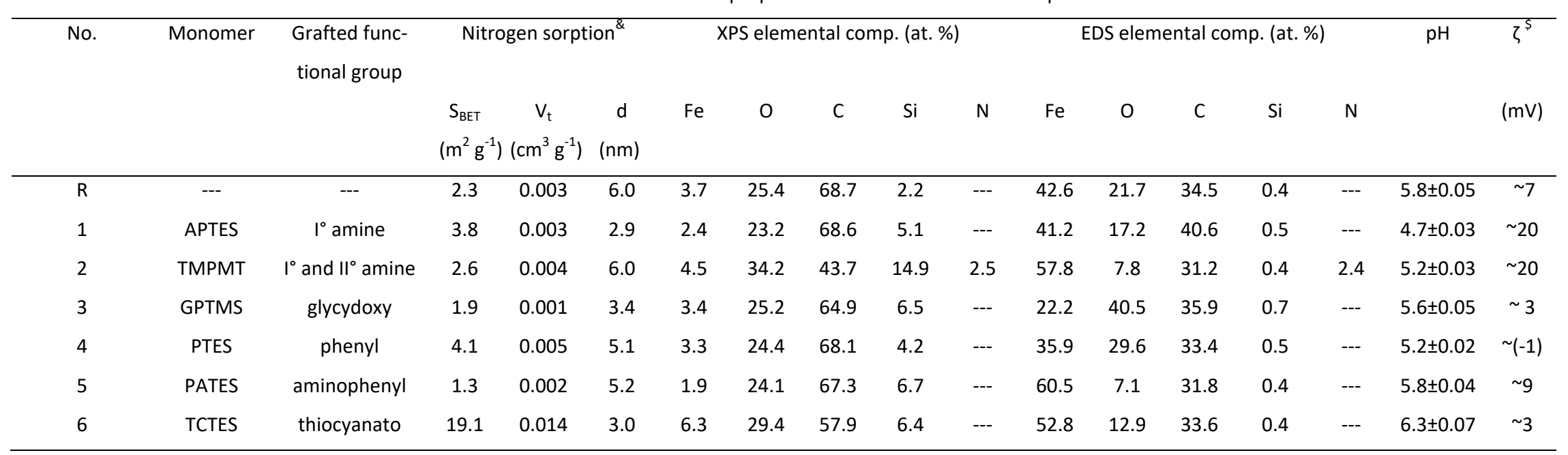

$261 \mathrm{~S}_{\mathrm{BET}}$ - specific surface area by BET method, $\mathrm{V}_{\mathrm{t}}$ - total volumes of the pores, $d$ - average pore size

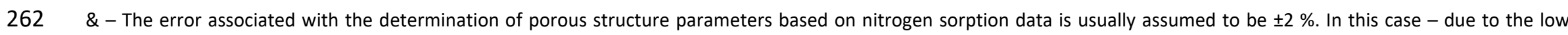
263 porosity - this error can be higher.

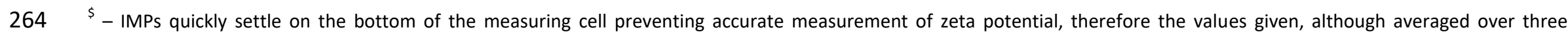
265 measurements, should be treated as approximate. 
The first visual observation is that the color of ethanol solution during the functionalization changes depending on the monomer used (Fig. S2b). This means that the outer silica layer of IMPs is not perfect and the solution containing organosilica monomers can be easily contacted with the carbonyl iron. Due to that some reactions can occur between the carbonyl iron and the organosilica monomers as well as traces of contaminants (note that the purity of the monomers is within the range of $95-97 \%$ so some initial reagents/co-products/catalysts are also present). Different colors of the solutions mean that

272 the different sets of reactions may occur. Interestingly, the reference sample R (no organosilica 273 monomer added) changed the color which means that some IMPs have nanometric dimensions and form 274 stable suspension of iron nanoparticles (cf. Fig 2b). Only the sample 2 does not change color what may 275 be explained by fast attachment of the silica monomer due to the presence of reactive methoxy groups. 276 In fact, high content of silica of the sample 2 (vide infra) is due to the formation of a tight organosilica 277 layer surrounding the IMPs, which does not allow the reactions, as is the case with other systems.

278 To investigate the effect of functionalization on the structural and chemical properties of the 279 resulting IMPs, they were submitted to thorough characterization by a wide range of instrumental 280 techniques. SEM images of the obtained microparticles are presented in Fig. 1. As can be seen the IMPs 281 are composed of spherical multisized and often agglomerated spheres. After functionalization the 282 particles remain unchanged - the only exception is sample 6, where complex formation/corrosion is 283 observed under higher magnification. Indeed, thiocyanates are considered to be highly corrosive to iron 284 and steel (Melendres, O'Leary, \& Solis, 1991; Ravald, Chilver, \& Williams, 2007) and they can also form 285 red complexes with iron. The occurring corrosion confirms the fact that the silica coating is not 286 sufficiently tight to protect the core iron from the contact with external environment. 


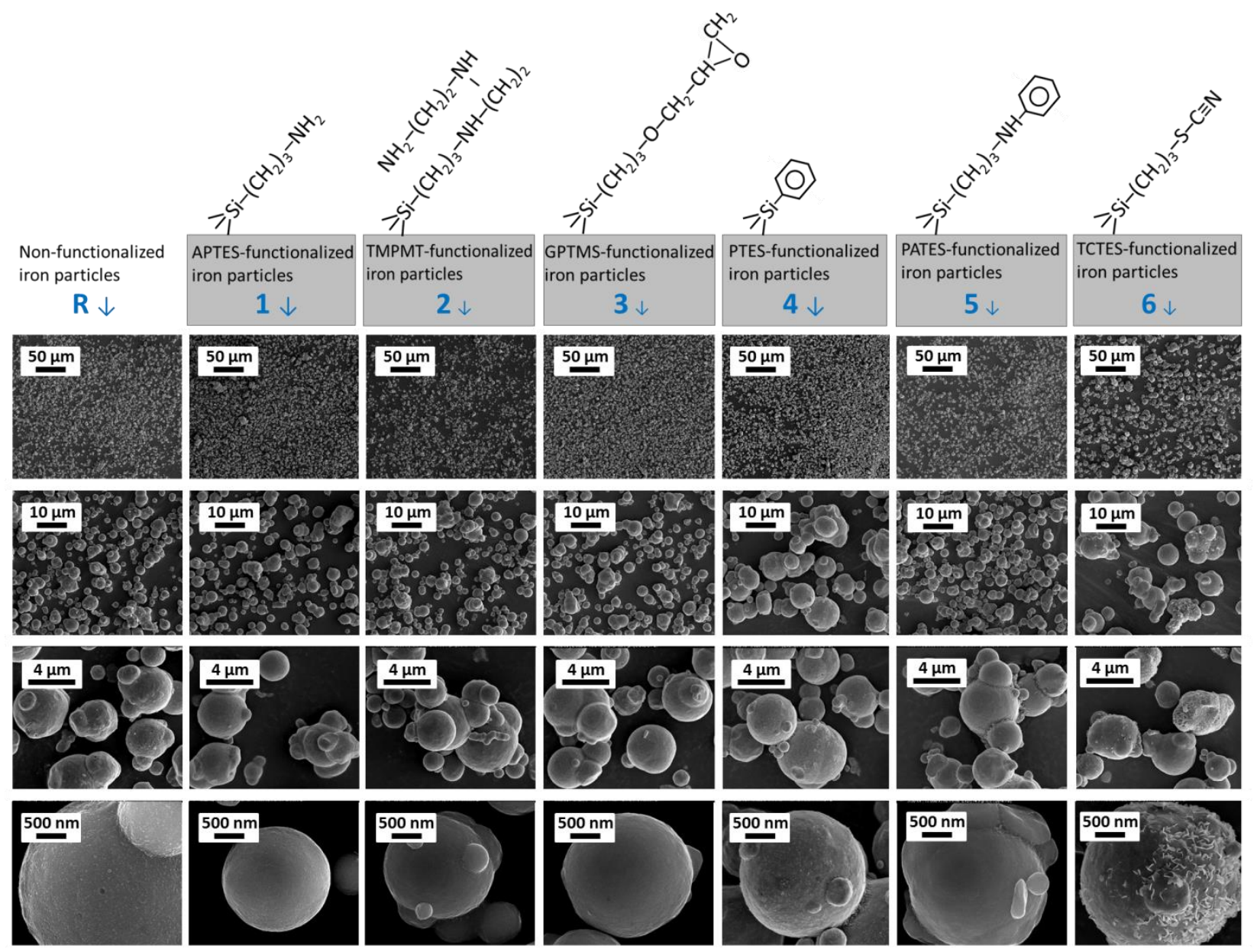

Fig. 1. SEM microphotographs of the iron microparticles at different magnifications

Structural parameters of the porous structure derived from nitrogen sorption isotherms are given

290 in Table 1. As can be seen all the microparticles but 6 have low specific surface areas (in the range of 1.3-

$2914.1 \mathrm{~m}^{2} \mathrm{~g}^{-1}$ ) and pore volumes (in the range 0.001-0.005 $\mathrm{cm}^{3} \mathrm{~g}^{-1}$ ). Only sample 6 has higher values of $\mathrm{S}_{\mathrm{BET}}$

292 and $V_{p}\left(19.1 \mathrm{~m}^{2} \mathrm{~g}^{-1}\right.$ and $0.014 \mathrm{~cm}^{3} \mathrm{~g}^{-1}$, respectively), when compared with the rest of the samples, due to

293 remarkable corrosion. The fast corrosion process of the sample 6 was confirmed by visual observation of

294 water solutions of microparticles in closed vials kept for 30 days (cf. Fig. S3). After that time reddish iron

295 oxide layer was formed in the case of 4 (after $\sim 10$ days) and 6 (after 1 day) but not in the case of the

296 remaining samples. This clearly testifies that the iron microparticles have different types of the external

297 layers, some of them inhibiting and some of them accelerating corrosion process. From the point of view

298 of biomedical applications the effective inhibition of corrosion is very important due to the permanent

299 contact of iron particles with different physiological fluids. Thus functional groups accelerating corrosion

300 cannot be used in those applications regardless of their functional usefulness. 
Apart from morphological features also the chemistry of the functionalized magnetic particles was thoroughly inspected by two quantitative methods: XPS and EDS. The first one is considered a surfacesensitive technique enabling determination of the surface composition to a depth up to several $\mathrm{nm}$ (Burrell, 2001) while the second - to several hundred nm (Prencipe, Dellasega, Zani, Rizzo, \& Passoni, 2015). When analyzing data collected in Table 1, the first conclusion is that the results collected using both techniques are significantly different which means that the chemical composition of the surface is different from the chemical composition of the bulk phase. Another general observation from the EDS elemental analysis (which was derived from three different probing regions for each sample) is that the overall chemical composition is extremely variable demonstrating the remarkable chemical heterogeneity (in addition to the structural one as evidenced by SEM analysis). For example looking at the EDS elements content it can be seen that the iron content is in the range 22.2-60.5\%, and oxygen 7.1-40.5 \%. The contents of carbon and silicon are more homogenous: 31.2-40.6\% and 0.4-0.7\%, respectively. In the case of the sample 2 functionalized by TMPET significant amount of nitrogen was detected by both, EDS (2.4\%) and XPS (2.5\%).

Interestingly, XPS elemental analysis shows that carbon is the more abundant element present on the surface, while the iron content is low. XPS elemental analysis reveals that the silicon content in the initial IMPs is only $2.2 \%$ while after each functionalization it elevates significantly, from $4.2 \%$ (sample 4) to $14.9 \%$ (sample 2). In the case of the latter the functionalization was the most efficient, which is also supported by the fact that the content of nitrogen introduced in the course of functionalization is high (2.4\%). In the case of other samples functionalized by monomers containing amine groups (samples 1 and 5), the amounts of nitrogen were apparently below the detection limit of both techniques.

The higher efficiency of functionalization with the use of TMPMT than APTES and PATES can be related to three factors. The first factor is that the larger ethoxy groups of APTES and PATES hydrolyse more slowly than smaller methoxy groups of MPTMS (Brochier Salon \& Belgacem, 2011; Osterholtz \& Pohl, 1992). The second factor is that the amine groups are additionally catalysing the processes of hydrolysis and condensation of TMPMT facilitating the formation of bigger clusters of co-condensed MPTMS molecules which are finally bound to the IMP' surface. The third factor is that metals and many metal oxides can strongly adsorb silanes if a chelating functionality such as diamine is present. The last two factors explain the difference in functionalization efficiency between TMPMT and GPTMS - another methoxy-derived monomer used in this work.

The values of surface $\mathrm{pH}$ and $\zeta$ potential also vary depending on the samples testifying to the remarkable chemical changes of the surface character occurring during functionalisation. In contrast to 
333 XPS and EDS techniques which have intrinsic local probing character, $\zeta$ potential and surface pH shows 334 overall effect of surface chemistry alterations. As can be seen in the case of the samples 1 and 2 the 335 values of $\zeta$ potential significantly shift towards more positive values, most probably due to the presence 336 of protonated amine groups. In contrast, for the sample 4 the value of $\zeta$ potential is slightly negative, 337 because of the presence of $\pi$ electrons of phenyl rings introduced during functionalization. Changes of $338 \mathrm{pH}$ values are more subtle; nevertheless, even those subtle changes show that the surface of magnetic 339 particles is different in each case. For example, lowering of $\mathrm{pH}$ from 5.8 for $\mathrm{R}$ sample to $4.7,5.2$ and 5.2 340 for the samples 1,2 and 5, respectively testify to the releasing $\mathrm{H}_{3} \mathrm{O}^{+}$ions from the protonated amine 341 groups. Majority of amine groups are protonated because during the functionalization (see Experimental 342 part for details) a small amount of hydrochloric acid was used to catalyze the hydrolysis of alkoxy groups 343 of the silica monomers.

\subsection{Preparation and structural characterization of magnetic alginate hydrogels}

Alginate hydrogels fabricated with the use of non-functionalized and functionalized iron microparticles were first inspected visually (Fig. 2). As can be seen they retained the shape of the container used for their preparation. All ferrogels were black, albeit with different macroscopic appearance: hydrogels 1, 2, and 5 were highly homogeneous, while in the case of the remaining samples, partial separation of water was observed on the next day (cf. Fig. 2: the water envelope around the hydrogels 3 and 4 is clearly visible). In the case of the hydrogel 6 the hydrogel structure was collapsed and the color was changed due to the formation of the red complexes with iron or/and progressing corrosion. Since all the hydrogels were obtained in the same time using the same stock solution of

354 alginate, the resulting changes in the physical appearance are undoubtedly attributed to the different 355 surface chemistries of the incorporated magnetic microparticles. For well-formed hydrogels (samples 1, 3562 and 5), magnetic microparticles were not only entrapped within the polymer network, but bonded 357 effectively to the alginate chains and consequently, no leakage of magnetic microparticles was observed 358 in these hydrogels (even after immersing samples 1, 2 and 5 in water for several days). On the contrary, as observed in Fig. 2, leakage of particles took place for the other hydrogels. 


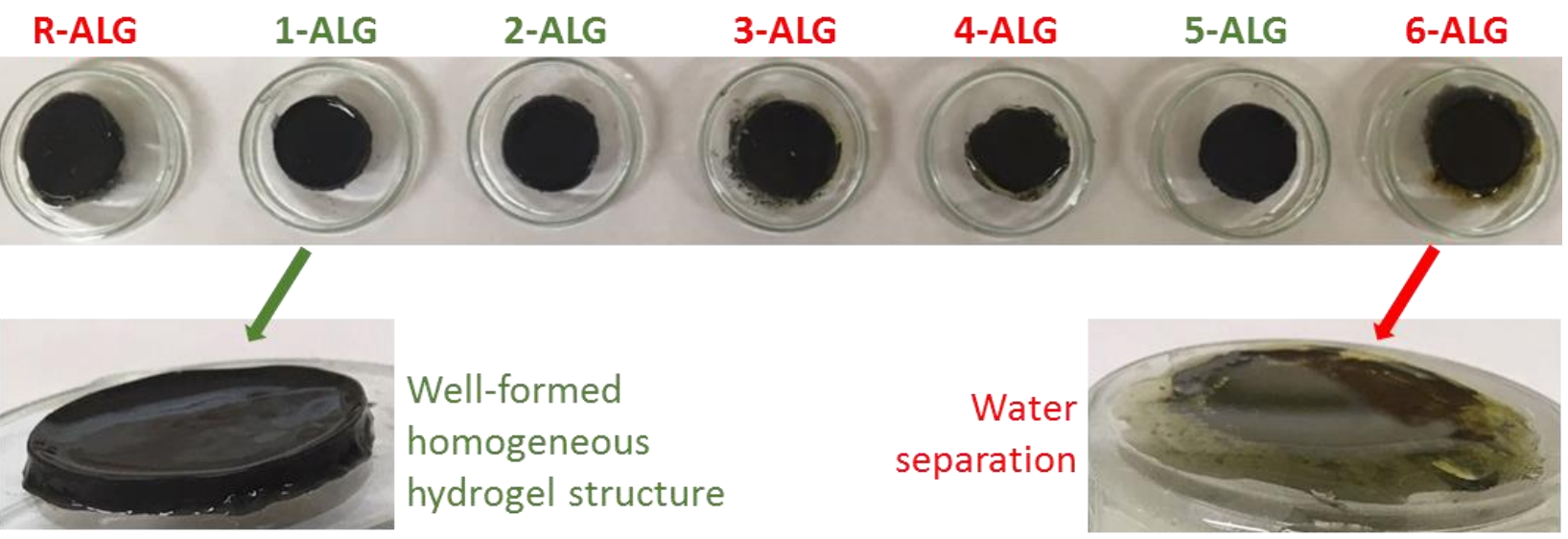

Fig. 2. Physical appearance of magnetic hydrogels studied. Note that in the case of the hydrogels 3,4 and 6 a liquid phase separated from the bulk hydrogel structure is seen

The water-releasing behavior of the magnetic hydrogels at room temperature is presented in Fig. 3a. For non-magnetic alginate hydrogel, A, the total weight loss was $98.4 \%$ while for the magnetic hydrogels the total weight losses were in the range $91.5-93.7 \%$. These small differences in the amount of the absorbed water suggest that the functionalization effect does not significantly affect the porosity of most hydrogels. However, statistical post hoc comparisons of 2-ALG vs 4-ALG pair and 4-ALG vs 6-ALG pair shows that the impact of surface functionalization can play an important role (cf. Fig. S6a). Water release rates are slightly different for different hydrogels and could be attributed to different surface chemistries of the magnetic microparticles. Statistical analysis shows, however, that the observed differences are not statistically significant. During the first 8 hours of water-release the slopes of the curves are similar for all the hydrogels because the bulk concentration of microparticles in all hydrogels is only $0.9 \%$, therefore most of the evaporating water during first hours of drying is the water absorbed in the pores of the hydrogel without direct contact with the surface of the iron microparticles. However, as the evaporation continues, more and more water molecules are in closer proximity to the functionalized surface of the microparticles. This is well seen in the last hours of drying, when some hydrogels are almost dry while others still hold water. Microparticles with primary amine groups (i.e., 1, 2) bind water more strongly than unmodified microparticles (R), or the ones modified with phenolic groups (4). This effect can be associated with various interactions of both specific (hydrogen bridges) and non-specific (electrostatic and hydrophobic interactions) character. These interactions may cause slower water release from the semi-dry hydrogel.

DSC thermograms of all tested hydrogels exhibit a broad endothermic peak starting at $\sim 100{ }^{\circ} \mathrm{C}$ (Fig. 3b), which indicates the loss of water from the hydrogel matrix. The fastest rate of water release is 
observed for R-ALG hydrogel, while for other hydrogels the dynamics of water evaporation is slower. This suggests that covering of iron particles with a silica layer, regardless of its functionalization, results in a more hydrophilic interphase in which water can be more strongly bound by the hydrophilic silica surface. Another reason may be related to differences in cross-linking of alginate, governed by various interactions of alginate chains with surface functionalities (vide infra). Although water evaporation peak maxima are clearly located at different temperatures, the results should be interpreted with caution as can strongly depend on the conditions of DSC experiments (Bellich, Borgogna, Carnio, \& Cesàro, 2009; Craig \& Reading, 2006). Nevertheless, the observed different DSC profiles (cf. Fig. 3b) confirm the water release observations of (cf. Fig. 3a), supporting the fact that water retention ability can be at least partly attributed to the effect of the presence of specific functional groups on the iron surface. In particular, the difference between hydrogels 1-ALG and 2-ALG and hydrogel 4-ALG is clearly visible. The overall conclusion can be summarized as follows: water is held more strongly by hydrogels with particles with hydrophilic surfaces (e.g. 1-ALG and 2-ALG) than by hydrogels with particles with hydrophobic surfaces (3-ALG and 5-ALG).

a

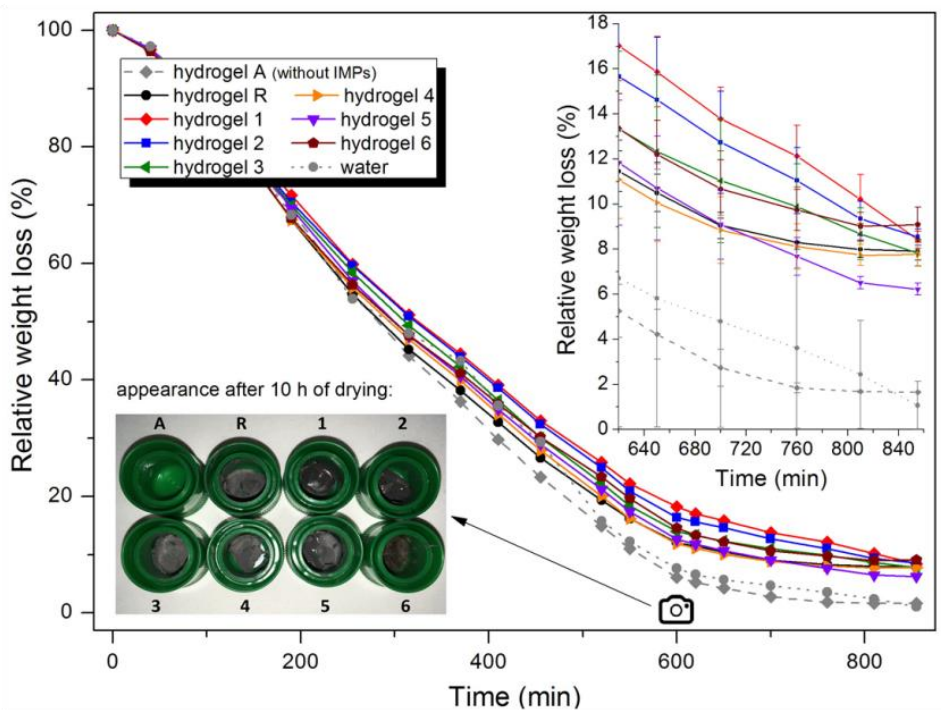

b

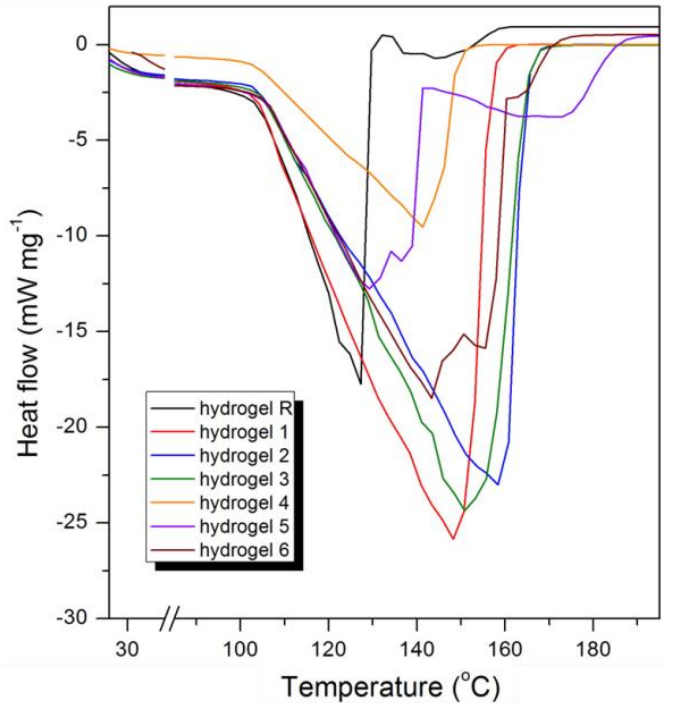

Fig. 3. (a) Water releasing kinetics upon drying (inlets: appearance of the magnetic hydrogels after 10 hours of drying (bottom), last period of drying, including error bars (top right), (b) DSC thermograms of wet magnetic hydrogels

In order to check whether the functionalization of microparticles affects the morphology of the final magnetic hydrogels, SEM imaging of selected hydrogels were run. The selected microphotographs are presented in Fig. 4 and a larger number of them is available in Supplementary. The pictures show that there are remarkable differences between ways of binding of the alginate hydrogels to the 
microparticles surface for the considered systems. In the case of R-ALG and 4-ALG, a characteristic cobweb-like network is visible which is connected only to the specific points of the surface of microparticles (marked with red circles on the Fig. 4). However, most of the surface is bare due to the lack of adequate surface chemistry that could ensure the appropriate interaction between alginate and iron microparticles. On the other hand, in the case of 1-ALG and 2-ALG hydrogels (i.e., hydrogels with abundance of amine groups on the surface), there is a completely different type of connectivity between both phases. The microparticles are surrounded by a hydrogel, without significant formation of cobweblike structures but rather tight covering of most of the microparticles' surface (marked with blue circles). In the case of the hydrogel 1-ALG, remarkable changes in the morphology of hydrogel chains surrounding some microparticles are seen, i.e., in some places alginate chains are clearly thicker probably due to strong attractive interactions between both phases.
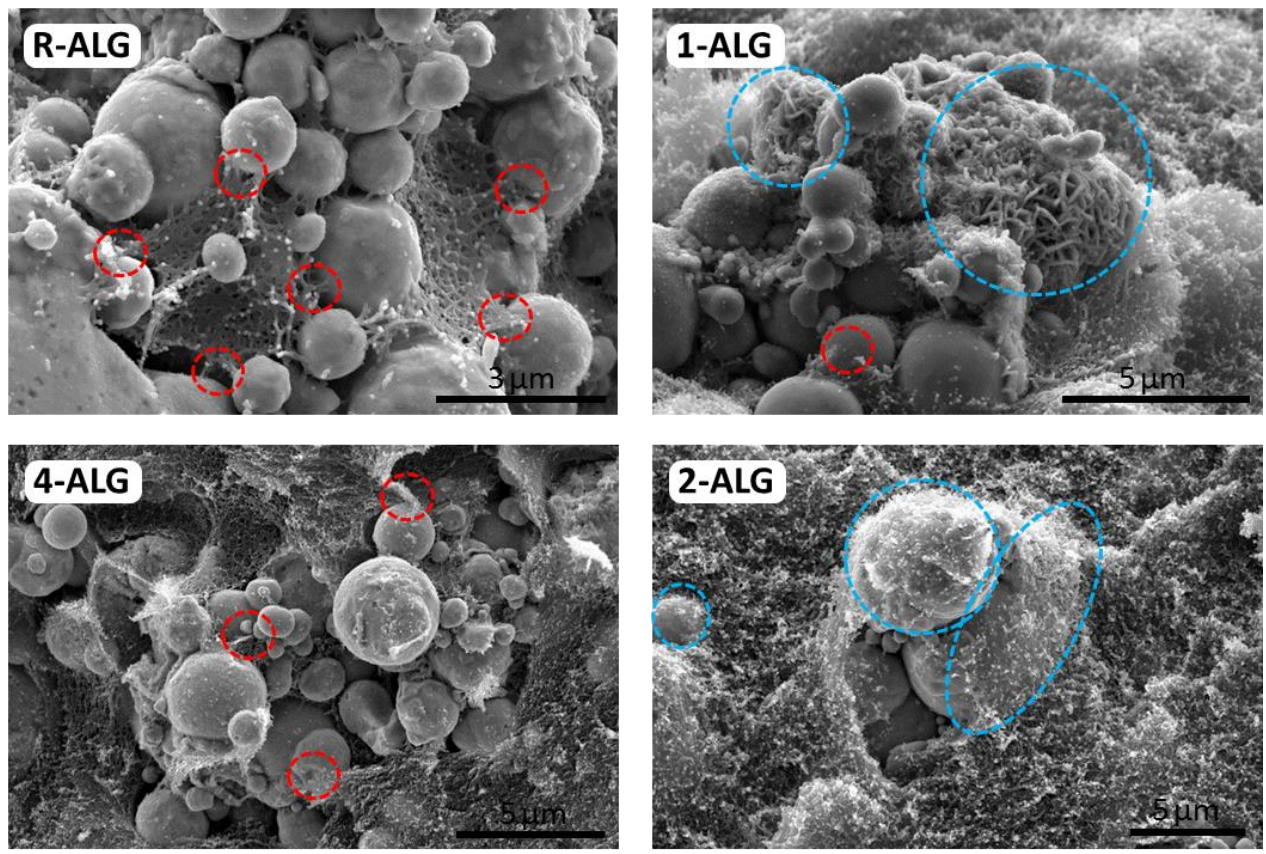

Fig. 4. SEM microphotographs of the selected hydrogels (red circles show cobweb-like single point type joints, blue circles multi point joints of the microparticles with alginate network)

\subsection{Quantum chemical description of possible interactions between alginate and functional groups}

To get more detailed view into the possible specific interactions of alginate with the surface groups (particularly amine groups) DFT calculations have been carried out. Calculations of the energetic effects accompanied sodium and calcium salt formation of alginic acid and comparison with those found for formation of complexes with functionalities present on the microparticle surface may give an insight into the competitive character of metal ions and functionalities interactions. It was found previously that 
the effect of presence of model silica surface the functionalities are attached to is not essential to the overall energetics (Barczak et al., 2018). Thus functionalities alone endcapped with hydrogen atoms were considered. The relevant formulas for calculating binding energies can be also found in our earlier papers

428 (Barczak \& Borowski, 2019; Barczak, Gil, Terpiłowski, Kamiński, \& Borowski, 2019; Barczak et al., 2018).

429 Calculations on systems like alginic acid sodium salt $[\mathrm{M}]_{m}[\mathrm{G}]_{n}$ (Fig. 5a), where $m$ and $n$ are fairly large, are 430 impractical unless very small basis sets or semi empirical methods are used. However, in such a case the 431 calculated energetics would be highly inaccurate. The alternative is to consider the case $m=n=1$ (the MG molecule) and perform the DFT calculations with extended basis set (cf. Computational details section).

433 The first problem we were faced with was endcapping of MG molecule, assumed to be a representative 434 fragment of the salt. There are two possibilities: termination with hydrogen atoms, i.e., formation of hydroxyl groups, or termination with methyl groups, i.e., formation of methoxy groups. The second choice (Fig. $5 b$ ) seems to be more appropriate one as in the $[\mathrm{M}]_{m}[\mathrm{G}]_{n}$ chain the bridging oxygen atoms are bound to $\mathrm{sp}^{3}$-hybridized carbon atom of a next unit ( $\mathrm{M}$ or $\mathrm{G}$ ). We believe that with such a simplified model the most important interactions with $\mathrm{Na}^{+}, \mathrm{Ca}^{2+}$, as well as with the functionalities attached to the nanoparticles will be accurately accounted for. The remaining systems representing sodium alginate considered in this work for the purpose of calculating relative energies are shown in Figs $5 c$ and $5 \mathrm{~d}$. The representative fragment of the calcium alginate is shown in Fig. $5 \mathrm{e}$. Note, that only one $\mathrm{Ca}^{2+}$ ion linking two chains was considered in the representative fragment to reduce the overall computational cost.

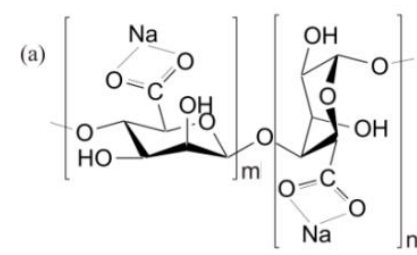

(M)

(G)

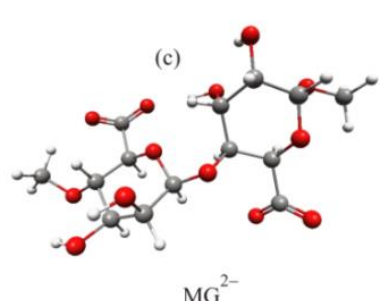

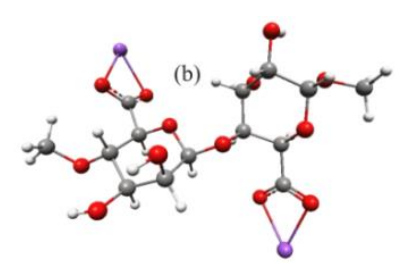

$\mathrm{MGNa}_{2}$

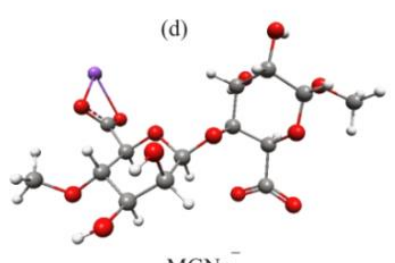

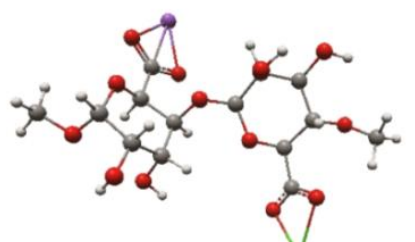

(e)

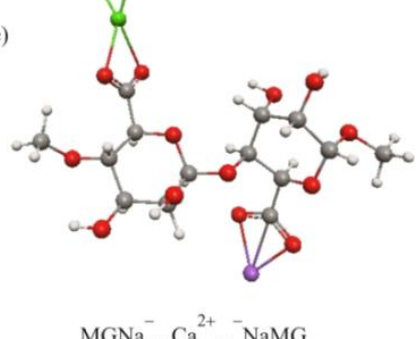

Fig. 5. (a) Sodium alginate monomer unit, (b) corresponding representative fragment endcapped with methoxy groups, $\mathrm{MGNa}_{2}$, (c) fully dissociated alginate unit, $\mathrm{MG}^{2-}$, (d) partially dissociated alginate unit, $\mathrm{MGNa}^{-}$, (e) two partially dissociated alginate units, $\mathrm{MGNa}^{-}$, cross-linked by $\mathrm{Ca}^{2+}$ cation. Colors of the atoms: grey - carbon, red - oxygen, white - hydrogen, violet - sodium, green - 
First the effect of dissociating of $\mathrm{Na}^{+}$cation from the starting model, $\mathrm{MGNa}_{2}$, was investigated (note that all energies are reported in $\mathrm{kJ} \mathrm{mol}^{-1}$ in the reaction schemes). Dissociation of the first and then 452 second sodium cation is associated with significant overall energy increase of $1298.6 \mathrm{~kJ} \mathrm{~mol}^{-1}$, i.e.,

$453 \quad \mathrm{MGNa}_{2} \stackrel{+573.8}{\longrightarrow} \mathrm{MGNa}^{-}+\mathrm{Na}^{+}$and $\mathrm{MGNa}^{-} \stackrel{+724.8}{\longrightarrow} \mathrm{MG}^{2-}+\mathrm{Na}^{+}$.

454 Such a high energy increase is associated with strong electrostatic interactions between oppositely 455 charged ions. Dissociation of $\mathrm{MGNa}^{-} \ldots \mathrm{Ca}^{2+} \ldots{ }^{-} \mathrm{NaMG}$ is even more energetically demanding, i.e.,

$456 \quad \mathrm{MGNa}^{-} \ldots \mathrm{Ca}^{2+} \ldots{ }^{-} \mathrm{NaMG} \stackrel{+1991.3}{\longrightarrow} 2 \mathrm{MGNa}^{-}+\mathrm{Ca}^{2+}$.

457 The binding energy of attaching the protonated amine group $\left(-\mathrm{NH}_{3}{ }^{+}\right)$to the carboxylic group $\left(-\mathrm{COO}^{-}\right)$ 458 accompanied by the formation of a complex shown in Fig. $6 a$ is nearly $-540 \mathrm{~kJ} \mathrm{~mol}^{-1}$,

$459 \quad \mathrm{MGNa}^{-}+{ }^{+} \mathrm{H}_{3} \mathrm{~N}-\mathrm{C}_{3} \mathrm{H}_{7} \stackrel{-534.9}{\longrightarrow} \mathrm{MGNa}^{-} \ldots{ }^{+} \mathrm{H}_{3} \mathrm{~N}-\mathrm{C}_{3} \mathrm{H}_{7}$.

460 It thus seems that in the case of sodium alginate favorable interactions between protonated amines and 461 carboxylic groups hardly occurs as it requires additional $38.9 \mathrm{~kJ} \mathrm{~mol}^{-1}$ (cf. Reaction 1 ). The situation seems 462 to be even less advantageous in the case of calcium alginate. However, the calculated energy of a 463 process

$464 \quad \mathrm{MGNa}^{-} \ldots \mathrm{Ca}^{2+} \ldots{ }^{-} \mathrm{NaMG} \stackrel{+549.2}{\longrightarrow} \mathrm{MGNa}^{-} \ldots \mathrm{Ca}^{2+}+\mathrm{MGNa}^{-}$

465 indicates that protonated amine groups may compete with calcium ions for carboxylic groups (this time 466 only extra $14.3 \mathrm{~kJ} \mathrm{~mol}^{-1}$ is required, cf. Reaction 3). On the other hand there is a number of other places 467 in the alginate monomer unit susceptible for attachment of protonated amine groups, like for example 468 structure shown in Fig. 6b. Note that protonated amine group is capable of forming two hydrogen bonds 469 with oxygen atoms present in the MG molecule: one is a bridging, and the other one is carboxylic 470 hydrogen atom. Such a double link should effectively stabilize the obtained complex. To calculate the 471 binding energies we used sodium alginate representative fragment (Fig. 5b) but the energies are not 472 expected to depend strongly on the chosen fragment. A few structures like those presented in Fig. 6b 473 were considered. All binding energies were found to be in the range from -146 to $-230 \mathrm{~kJ} \mathrm{~mol}^{-1}$. These 474 values are well below $-80 \mathrm{~kJ} \mathrm{~mol}^{-1}$, which means that the complexes are very stable at room 475 temperature. The less negative binding energy (indicating that the complex is least stable) was found for 476 the system in which the double link involves two "ether-like" oxygen atoms (e.g., bridging and hydroxyl 477 hydrogen atoms). The most negative binding energies were obtained when one of the oxygen atoms was 478 carboxylic oxygen. These energies are large enough for the complex to be very stable at room 479 temperature and follow from strong ion-dipole interactions $\left(U^{\text {int }} \sim r^{-2}\right)$. It thus appears that, regardless 
of the accessibility of carboxylic groups to functionalities present on the nanoparticle surface, alginate acid salts can be successfully bound to the aminated surface of iron particles. As discussed earlier, this is because the alginate chains can interact with specific functionalities also via not-carboxylic oxygen arrangements, as shown in Fig. 6b.

More interesting is the case of protonated $-\mathrm{S}-\mathrm{C} \equiv \mathrm{N}$ groups (denoted $-\mathrm{SCN}^{+} \mathrm{H}$ ). The structures considered are shown in Figs $6 \mathrm{c}$ and $\mathrm{d}$. The binding energy in a following process (Fig. $6 \mathrm{c}$ )

$$
\mathrm{MGNa}^{-}+\mathrm{H}^{+} \mathrm{NCS}-\mathrm{C}_{3} \mathrm{H}_{7} \stackrel{-614.8}{\longrightarrow} \mathrm{MGNa}^{-} \ldots \mathrm{H}^{+} \mathrm{NCS}-\mathrm{C}_{3} \mathrm{H}_{7}
$$

487 is high. The $-\mathrm{SCN}^{+} \mathrm{H}$ group seems to compete successfully with metal ions for the carboxylic group (cf. 488 energies reported in Reaction 1 and 4). In addition, $-\mathrm{SCN}^{+} \mathrm{H}$ group can be bound to a variety of noncarboxylic oxygen atoms (similarly to the protonated amine group). One of the structures found is shown in Fig. $6 \mathrm{~d}$ and the binding energy is equal to $204.9 \mathrm{~kJ} \mathrm{~mol}^{-1}$. Unfortunately the SCN-modified microparticles (sample 6) are strongly corroded (cf. Figs 2 and S3) thus we could not fully verify their effect on the final properties of magnetic hydrogels. However, in the case of other non-corroding particles (like for example silica, titania or carbon nano/microparticles) the strong interactions predicted by our calculations can be easily verified.
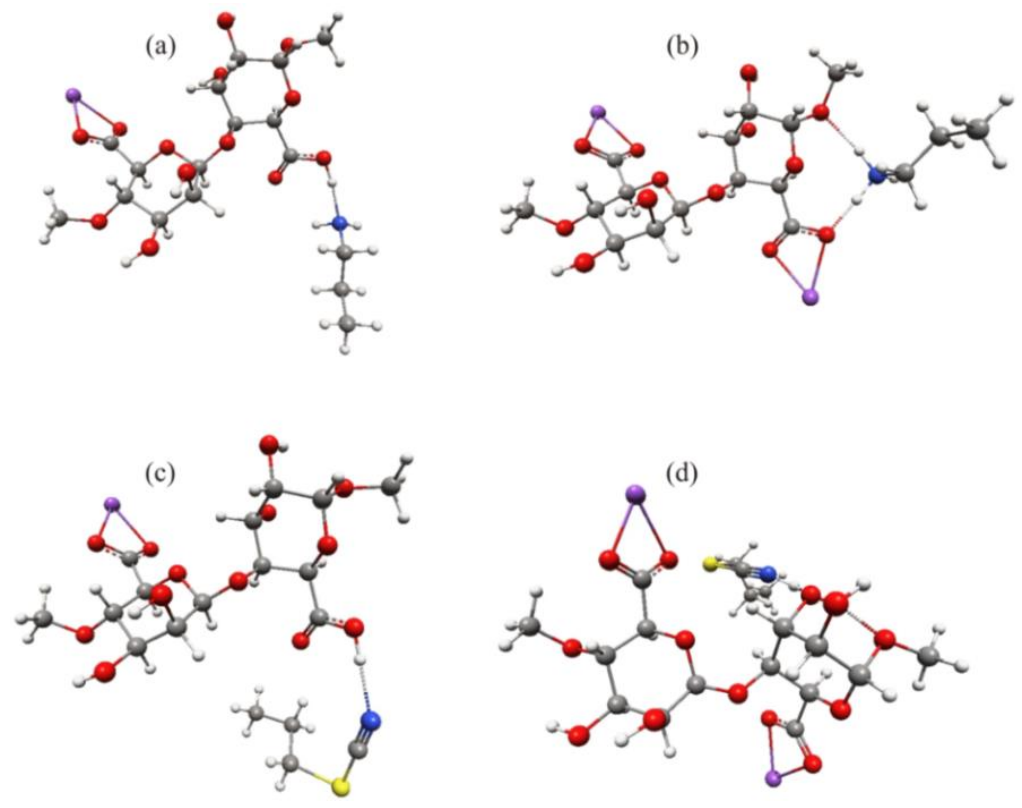

Fig. 6. (a) Complex between protonated amine group and $\mathrm{MGNa}^{-}$involving carboxylic group, (b) $\mathrm{MGNa}_{2}$ doubly linked to the protonated amine group, (c) complex between protonated $-\mathrm{S}-\mathrm{C} \equiv \mathrm{N}$ group and $\mathrm{MGNa}^{-}$involving carboxylic group, (d) $\mathrm{MGNa}_{2}$ linked to the protonated $-\mathrm{S}-\mathrm{C} \equiv \mathrm{N}$ group through hydroxyl oxygen. Colors of the atoms: grey - carbon, red - oxygen, white - 
Rheological characteristics of all the hydrogels but ALG-6 were tested using well-known protocols adopted by us in our previous works. The dependence of the storage modulus ( $\left.G^{\prime}\right)$ and loss modulus ( $\left.G^{\prime \prime}\right)$ as a function of the shear strain amplitude, SSA, in oscillatory regime $(v=1 \mathrm{~Hz})$ is shown in Fig. S4 -as an example, result for R-ALG hydrogel is shown in Fig 7a too. It has a typical shape for a viscoelastic solidlike material characterized by $G^{\prime}>G^{\prime \prime}$ at low strain amplitude (Gila-Vilchez et al., 2018). This means that the hydrogel exhibits a solid-like response. Both viscoelastic moduli have a broad plateau-like region within the range of $\sim 0.01-5 \%$ of SSA, which is called linear viscoelastic region (LVR). When SSAs reach critical values, values of $G^{\prime}$ decrease dramatically, whereas $G^{\prime \prime}$ first increase reaching maximum and decrease afterwards. The increase in $G^{\prime \prime}$ represents an enhancement in the dissipation of energy related to the irreversible destruction of the microstructure of the hydrogel by the shear forces. As observed in Fig. S4, this maximum in G' approximately coincides with the intersection of the curves of $G^{\prime}$ and $G^{\prime \prime}$, with $G^{\prime \prime}$ being higher than $G^{\prime}$ above this maximum point, which represents a liquid-like behavior. This region where $G^{\prime}$ and $G^{\prime \prime}$ experience rapid changes in their magnitude is known as nonlinear viscoelastic region (NVR). Within this region, irreversible deformation of the internal structure of the hydrogels occurs, which results in the observed decrease of elasticity manifested by huge decrease of $G^{\prime}$. At the microscopic level, these changes are explained by increasing friction between the hydrogel chains/segments (increase in loss modulus, $G^{\prime \prime}$ ) as well as possible breakage of the alginate segments (Cvek et al., 2020). The observed differences in G' values between hydrogels are significant for 1-ALG, 2ALG and 5-ALG hydrogels compared to R-ALG (Dunnett post hoc test, $\alpha=0.05$ ) with $p$-values respectively $0.0001,0.001$ and 0.044 . However, for 3-ALG and 4-ALG hydrogels, no significant difference was seen compared to R-ALG. Detailed statistical analysis was included in Supplementary data (cf. Fig. S6b).

Looking at these values collected in Table 2 (and also, for better visualization in Fig. 7c) it can be seen that the incorporation of amine-modified microparticles 1 and 2 results in much higher values of $\mathrm{G}^{\prime}$ of the resulting hydrogels 1-ALG and 2-ALG (410 and $483 \mathrm{~Pa}$, respectively) when compared with the hydrogel R-ALG (137 Pa). In contrast, hydrogels doped with glycidoxy- and phenyl-functionalized IMPs (3 and 4, respectively) have almost non-affected $\mathrm{G}^{\prime}$ values (112 and $141 \mathrm{~Pa}$, respectively). These results agree with the differences in adhesion between polymer chains and particles observed by means of electron microscopy (Fig. 4), and clearly demonstrate the critical role of the proper functionalization of the iron particles in governing final mechanical properties of the resulting hydrogels. Another characteristic feature is the maximum in $\mathrm{G}^{\prime \prime}$ modulus that is known as yielding point, at which dissipation of energy is maximal (Moghimi, Jacob, Koumakis, \& Petekidis, 2017). The values of SSA corresponding to 
533 the maximal values of G" are also collected in Table 2. Differences between them can be also attributed

534 to the changes of the structure of hydrogels due to different interactions with various microparticles.

Table 2. Mean values of G' corresponding to the LVR and values of SSA in yielding point for the hydrogels studied

\begin{tabular}{|c|c|c|c|c|c|c|}
\hline \multirow[b]{2}{*}{ hydrogel } & \multicolumn{2}{|c|}{ No magnetic field } & \multicolumn{2}{|c|}{ Magnetic field: $141 \mathrm{kA} \mathrm{m}^{-1}$} & \multicolumn{2}{|c|}{ Magnetic field: $282 \mathrm{kA} \mathrm{m}^{-1}$} \\
\hline & $\begin{array}{l}\mathrm{G}_{\text {LVR }}^{\prime} \\
(\mathrm{Pa})\end{array}$ & $\begin{array}{c}\text { SSA of } \mathrm{G}^{\prime \prime}{ }_{\text {max }} \\
(\%)\end{array}$ & $\begin{array}{l}\mathrm{G}_{\text {LVR }}^{\prime} \\
(\mathrm{Pa})\end{array}$ & $\begin{array}{c}\text { SSA of } G_{\text {max }}^{\prime \prime} \\
(\%)\end{array}$ & $\begin{array}{l}\mathrm{G}_{\mathrm{LVR}}^{\prime} \\
(\mathrm{Pa})\end{array}$ & $\begin{array}{c}\text { SSA of } G^{\prime \prime}{ }_{\text {max }} \\
(\%)\end{array}$ \\
\hline R-ALG & $137 \pm 34$ & $5-26$ & $1278 \pm 151$ & 72 & $2088 \pm 202$ & 36 \\
\hline 1-ALG & $410 \pm 105$ & 18 & $1429 \pm 243$ & 43 & $2117 \pm 338$ & 36 \\
\hline $2-A L G$ & $483 \pm 83$ & 22 & $1600 \pm 33$ & 51 & $2267 \pm 75$ & 43 \\
\hline 3-ALG & $112 \pm 22$ & $13-72$ & $1486 \pm 357$ & 51 & $2297 \pm 441$ & 31 \\
\hline 4-ALG & $141 \pm 24$ & 16 & $1223 \pm 250$ & 43 & $1982 \pm 373$ & 36 \\
\hline 5-ALG & $286 \pm 58$ & $13-36$ & $1152 \pm 404$ & 43 & $1660 \pm 661$ & 31 \\
\hline
\end{tabular}

536
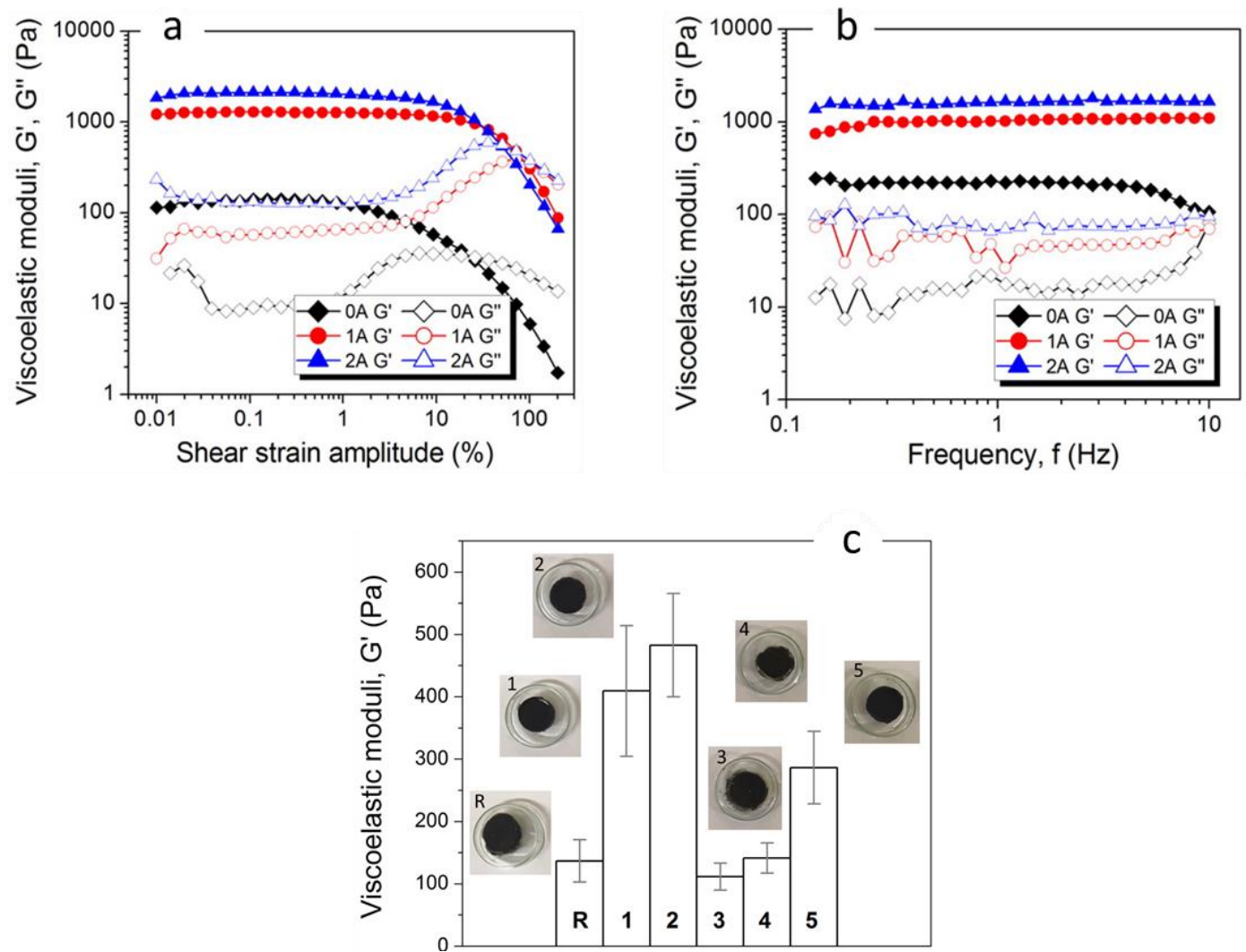

Fig. 7. (a) Storage and loss moduli of the R-ALG hydrogel as a function of shear strain amplitude. (b) Storage and loss moduli of R-ALG hydrogel as a function of frequency. OA refers to the measurements without the presence of magnetic field, $1 \mathrm{~A}$ and $2 \mathrm{~A}$

540 refer to the magnetic field of $141 \mathrm{kA} \mathrm{m-1}$ and $242 \mathrm{kA} \mathrm{m-1}$, respectively. (c) Comparison of the values of storage modulus (G') of 
In the presence of the magnetic field strong increase of the viscoelastic moduli is observed due to the magnetically induced reorganization of the structure, which was not hindered by the elastic matrix of alginate hydrogel. Furthermore, as observed in Fig. S4 and Table 2 (see also Fig. 7a for R-ALG hydrogel), in the presence of applied magnetic field the onset of the NVR and the yielding point move in general to higher values of the SSA, with respect to the absence of applied field, something that must be connected to the strengthening of the microstructure due to the interparticle attraction mediated by the applied magnetic field. The magnetic field-dependence for all the magnetic hydrogels is similar.

The dependence of both viscoelastic moduli as a function of frequency within the LVR was also analyzed and is presented in Fig. S5 (as an example, result for R-ALG hydrogel is shown in Fig 7b too). As observed, both moduli, $G^{\prime}$ and $G^{\prime \prime}$, only slightly change with the frequency of oscillation for the range of frequencies under study $(0.1-10 \mathrm{~Hz})$. In all cases $\mathrm{G}^{\prime}$ was considerably larger than $\mathrm{G}^{\prime \prime}$. The observed tendencies are typical of cross-linked polymer systems (Macosko, 1994), as well as of soft human tissues (Callejas et al., 2017). As expected, the values of $G^{\prime}$ and $G^{\prime \prime}$ remarkably increases when magnetic field is applied during rheological measurements.

\subsection{Assessment of cell viability}

Finally, cell viability of the selected hydrogels has been tested. At this point it should be mentioned that we wanted merely to compare the selected hydrogels (i.e., R-ALG, 2-ALG and 4-ALG) with each other rather than to assess absolute cytotoxicity. This is due to the fact that a thorough cytotoxicological study would require finding interrelations between the cell viability and alginate concentration, cell density, calcium concentration and exposing time. For example, it was reported that exposure of cells to the calcium environment (note that calcium ions are involved in crosslinking process) can cause significant loss of living cells in culture media (Cao, Chen, \& Schreyer, 2012). Such as rigorous study was not the aim of this work. Representative fluorescence micrographs of calcein-AM-stained, live cells (green) and propidium iodide-stained, dead cells (red), corresponding to the Live/Dead assay are shown in Fig. 8a. As can be seen, the number of live cells was significantly reduced for all alginate magnetic hydrogels studied when compared with the control cells. Most importantly, we observed differences between the tested hydrogels: in the case of R-ALG sample, no dead cells were observed on the top of the hydrogel, while in the case of 4-ALG sample, a significant number of dead cells was found on some images. Statistical analysis of the functional WST-1 assays (Fig. 8b) showed significant differences among

572 hydrogels R-ALG and 4-ALG (p-value<0.0001) but not R-ALG and 2-ALG ( $p$-value=0.016). This means that 573 amine groups present on the surface of the sample 2 does not results in decreased cell viability. In 
574 contrast, functionalization with phenyl groups (sample 4) makes the resulting hydrogel less 575 biocompatible. Therefore, it can be concluded that biocompatibility of the hydrogels depends on the 576 surface chemistry of functionalized microparticles and thus, apart from the macroscopic and mechanical 577 properties, also the cell viability depends on the functionalization (cf. Fig. S6c). These results are 578 confirmed by the free DNA quantification analysis showing statistically significant differences between all 579 the compared samples (cf. Fig. S6d). As it can be seen from the Fig. 8c, 4-ALG hydrogel was associated 580 with the highest levels of cell damage and DNA release to the culture medium, while the hydrogels R581 ALG and 2-ALG showed much better biocompatibility.
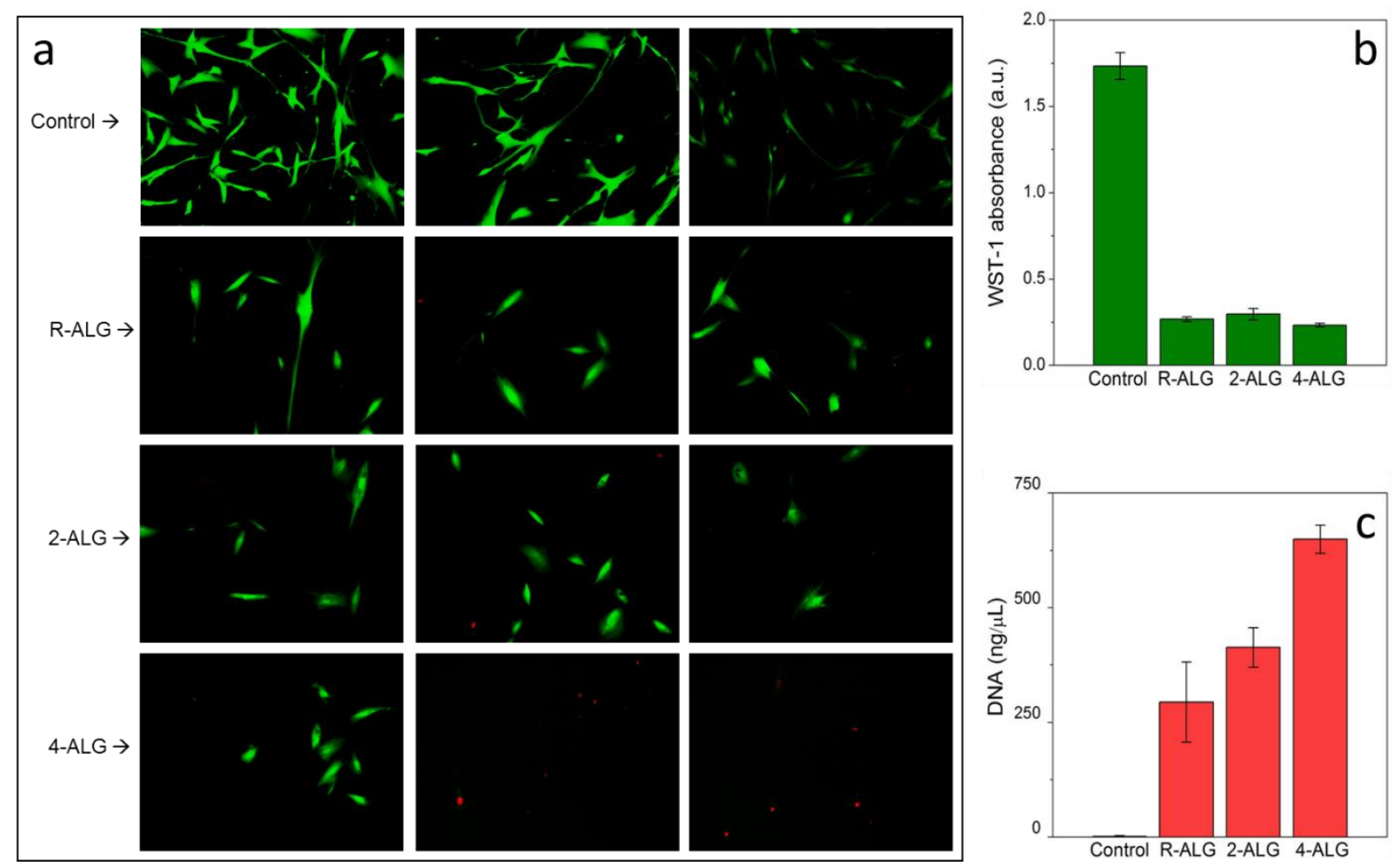

Fig. 8. (a) Cytotoxicity of the selected hydrogels revealed by the fluorescence microscopy, (b) WST-1 absorbance test, (c) and DNA quantification in the cell medium

\section{Conclusions}

Iron microparticles were modified by introducing a number of functionalities on their surface, ranging 588 from amine to phenyl groups. Although surface functionalizations have not significantly affected the 589 properties of the microparticles themselves, they changed remarkably the final properties of magnetic 590 hydrogels obtained by embedding the iron microparticles into the pre-polymerized alginate matrix. Thus, 591 the successful dispersion of functionalized microparticles was twofold beneficial: (i) magnetic activity 
was introduced in-situ, (ii) enhancement of the macroscopic and mechanical properties was achieved thanks to the altered interactions of alginate with functionalized surface. Among all the systems studied, amine functionalized IMP-based hydrogels exhibited superior properties when compared with the hydrogel prepared with the use of their non-functionalized counterpart. Properties such as hydrogel integrity, water-holding capability, storage modulus of the amine-based hydrogels of 2-ALG and 4-ALG were significantly altered in comparison with R-ALG hydrogel. For example, storage moduli for the former ones are 410 and $483 \mathrm{~Pa}$, respectively, while for the latter - only $137 \mathrm{~Pa}$. SEM images revealed that the lack of adequate surface chemistry limits the contact between both phases, which are connected only by limited number of anchoring points. In contrast, amination of the iron surface results in more tight covering of most of the microparticles' surface by multiple connections. Theoretical DFT calculations revealed that alginate chains are chemically active not only because of the presence of carboxyl groups but also other non-carboxylic oxygen arrangements which can interact with functionalities. Even blocking of all carboxyl groups by calcium cations during alginate crosslinking does not limit the possibility of tuning of alginate interactions with appropriately modified surfaces.

\section{Acknowledgements}

Mariusz Barczak thanks to Polish National Agency for Academic Exchange (NAWA) for supporting the research and his stay at the University of Granada in the frame of the Bekker programme fellowship no. PPN/BEK/2018/1/00235/U/00001. This study was supported by project FIS2017-85954-R (Ministerio de Economía, Industria y Competitividad, MINECO, and Agencia Estatal de Investigación, AEI, Spain, cofunded by Fondo Europeo de Desarrollo Regional, FEDER, European Union).

\section{Literature}

Anderson, T. F. (1951). Techniques for the preservation of three-dimensional structure in preparing specimens for the electron microscope. New York Academy of Sciences. Transactions, 13, 130-134.

Baker, J., Wolinski, K., Malagoli, M., Kinghorn, D., Wolinski, P., Magyarfalvi, G., ... Pulay, P. (2009). Quantum chemistry in parallel with PQS. Journal of Computational Chemistry, 30(2), 317-335. https://doi.org/10.1002/jcc.21052

Barbucci, R., Giani, G., Fedi, S., Bottari, S., \& Casolaro, M. (2012). Biohydrogels with magnetic nanoparticles as crosslinker: Characteristics and potential use for controlled antitumor drug- 
delivery. Acta Biomaterialia, 8(12), 4244-4252. https://doi.org/10.1016/J.ACTBIO.2012.09.006

624

625

626

627

628

629

630

631

632

633

634

635

636

637

638

639

640

641

642

643

644

645

646

647

648

649

650

651

652

653

654

Barczak, M., \& Borowski, P. (2019). Silica xerogels modified with amine groups: Influence of synthesis parameters on porous structure and sorption properties. Microporous and Mesoporous Materials, 281, 32-43. https://doi.org/10.1016/J.MICROMESO.2019.02.032

Barczak, M., Gil, M., Terpiłowski, K., Kamiński, D., \& Borowski, P. (2019). Influence of bridged monomer on porosity and sorption properties of mesoporous silicas functionalized with diethylenetriamine groups. Adsorption, 25(3), 575-589. https://doi.org/10.1007/s10450-019-00047-z

Barczak, M., Wierzbicka, M., \& Borowski, P. (2018). Sorption of diclofenac onto functionalized mesoporous silicas: Experimental and theoretical investigations. Microporous and Mesoporous Materials, 264, 254-264. https://doi.org/10.1016/J.MICROMESO.2018.01.013

Bellich, B., Borgogna, M., Carnio, D., \& Cesàro, A. (2009). Thermal behavior of water in micro-particles based on alginate gel. In Journal of Thermal Analysis and Calorimetry (Vol. 97, pp. 871-878). Springer. https://doi.org/10.1007/s10973-009-0392-x

Bonhome-Espinosa, A. B., Campos, F., Rodriguez, I. A., Carriel, V., Marins, J. A., Zubarev, A., ... LopezLopez, M. T. (2017). Effect of particle concentration on the microstructural and macromechanical properties of biocompatible magnetic hydrogels. Soft Matter, 13(16), 2928-2941. https://doi.org/10.1039/C7SM00388A

Brochier Salon, M.-C., \& Belgacem, M. N. (2011). Hydrolysis-Condensation Kinetics of Different Silane Coupling Agents. Phosphorus, Sulfur, and Silicon and the Related Elements, 186(2), 240-254. https://doi.org/10.1080/10426507.2010.494644

Buenger, D., Topuz, F., \& Groll, J. (2012). Hydrogels in sensing applications. Progress in Polymer Science, 37(12), 1678-1719. https://doi.org/10.1016/J.PROGPOLYMSCI.2012.09.001

Burrell, M. C. (2001). Chemical Analysis, Electron Spectroscopy. Encyclopedia of Materials: Science and Technology, 1142-1149. https://doi.org/10.1016/B0-08-043152-6/00214-X

Callejas, A., Gomez, A., Melchor, J., Riveiro, M., Massó, P., Torres, J., ... Rus, G. (2017). Performance study of a torsional wave sensor and cervical tissue characterization. Sensors (Switzerland), 17(9). https://doi.org/10.3390/s17092078

Čampelj, S., Makovec, D., \& Drofenik, M. (2009). Functionalization of magnetic nanoparticles with 3aminopropyl silane. Journal of Magnetism and Magnetic Materials, 321(10), 1346-1350. https://doi.org/10.1016/j.jmmm.2009.02.036

Cao, N., Chen, X. B., \& Schreyer, D. J. (2012). Influence of Calcium Ions on Cell Survival and Proliferation in the Context of an Alginate Hydrogel. International Scholarly Research Network ISRN Chemical 
Censi, R., Gigliobianco, M. R., Malaj, L., \& Di Martino, P. (2016). Effect of poly(vinylpyrrolidone) or sodium alginate on the stability of the amorphous form of nimesulide. Journal of Thermal Analysis and Calorimetry, 123(3), 2415-2425. https://doi.org/10.1007/s10973-015-5175-y

Craig, D. Q. M., \& Reading, M. (2006). Thermal Analysis of Pharmaceuticals. Thermal Analysis of

Pharmaceuticals. CRC Press. https://doi.org/10.1201/9781420014891

Cvek, M., Zahoranova, A., Mrlik, M., Sramkova, P., Minarik, A., \& Sedlacik, M. (2020). Poly(2-oxazoline)based magnetic hydrogels: Synthesis, performance and cytotoxicity. Colloids and Surfaces B: Biointerfaces, 190, 110912. https://doi.org/10.1016/j.colsurfb.2020.110912

De Groot, C. J., Van Luyn, M. J. ., Van Dijk-Wolthuis, W. N. ., Cadée, J. A., Plantinga, J. A., Otter, W. Den, \& Hennink, W. E. (2001). In vitro biocompatibility of biodegradable dextran-based hydrogels tested with human fibroblasts. Biomaterials, 22(11), 1197-1203. https://doi.org/10.1016/S01429612(00)00266-0

Dimatteo, R., Darling, N. J., \& Segura, T. (2018). In situ forming injectable hydrogels for drug delivery and wound repair. Advanced Drug Delivery Reviews, 127, 167-184. https://doi.org/10.1016/J.ADDR.2018.03.007

Donati, I., Gamini, A., Skjåk-Bræk, G., Vetere, A., Campa, C., Coslovi, A., \& Paoletti, S. (2003). Determination of the diadic composition of alginate by means of circular dichroism: A fast and accurate improved method. Carbohydrate Research, 338(10), 1139-1142. https://doi.org/10.1016/S0008-6215(03)00094-6

Drury, J. L., \& Mooney, D. J. (2003). Hydrogels for tissue engineering: scaffold design variables and applications. Biomaterials, 24(24), 4337-4351. https://doi.org/10.1016/S0142-9612(03)00340-5

Du, X., Zhou, J., Shi, J., \& Xu, B. Supramolecular Hydrogelators and Hydrogels: From Soft Matter to Molecular Biomaterials, 115 Chemical Reviews $\S(2015)$. American Chemical Society. Retrieved from https://pubs.acs.org/doi/10.1021/acs.chemrev.5b00299

Espona-Noguera, A., Ciriza, J., Cañibano-Hernández, A., Fernandez, L., Ochoa, I., Saenz del Burgo, L., \& Pedraz, J. L. (2018). Tunable injectable alginate-based hydrogel for cell therapy in Type 1 Diabetes Mellitus. International Journal of Biological Macromolecules, 107, 1261-1269. https://doi.org/10.1016/J.IJBIOMAC.2017.09.103

Fan, D., Tian, Y., \& Liu, Z. (2019). Injectable Hydrogels for Localized Cancer Therapy. Frontiers in Chemistry, 7, 675. https://doi.org/10.3389/fchem.2019.00675

Filippov, M. P., \& Kohn, R. (1974). Determination of composition of alginates by infrared spectroscopic 
method. Chemicke Zvesti, 28(6), 817-819.

Fitzpatrick, S. E., Staiger, M. P., Deb-Choudhury, S., \& Ranford, S. (2018). Protein-based Aerogels: Processing and Morphology. In RSC Green Chemistry (Vol. 2018-Janua, pp. 67-102). Royal Society of Chemistry. https://doi.org/10.1039/9781782629979-00067

Geckil, H., Xu, F., Zhang, X., Moon, S., \& Demirci, U. (2010). Engineering hydrogels as extracellular matrix mimics. Nanomedicine, 5(3), 469-484. https://doi.org/10.2217/nnm.10.12

Giani, G., Fedi, S., \& Barbucci, R. (2012). Hybrid Magnetic Hydrogel: A Potential System for Controlled Drug Delivery by Means of Alternating Magnetic Fields. Polymers, 4(2), 1157-1169. https://doi.org/10.3390/polym4021157

Gila-Vilchez, C., Bonhome-Espinosa, A. B., Kuzhir, P., Zubarev, A., Duran, J. D. G., \& Lopez-Lopez, M. T. (2018). Rheology of magnetic alginate hydrogels. Journal of Rheology, 62(5), 1083-1096. https://doi.org/10.1122/1.5028137

Gila-Vilchez, C., Duran, J. D. G., Gonzalez-Caballero, F., Zubarev, A., \& Lopez-Lopez, M. T. (2019). Magnetorheology of alginate ferrogels. Smart Materials and Structures, 28(3), 035018. https://doi.org/10.1088/1361-665X/aafeac

Gila-Vilchez, C., Mañas-Torres, M. C., Contreras-Montoya, R., Alaminos, M., Duran, J. D. G., de Cienfuegos, L. Á., \& Lopez-Lopez, M. T. (2019). Anisotropic magnetic hydrogels: design, structure and mechanical properties. Philosophical Transactions of the Royal Society A: Mathematical, Physical and Engineering Sciences, 377(2143), 20180217. https://doi.org/10.1098/rsta.2018.0217

Griffin, D. R., Weaver, W. M., Scumpia, P. O., Di Carlo, D., \& Segura, T. (2015). Accelerated wound healing by injectable microporous gel scaffolds assembled from annealed building blocks. Nature Materials, 14(7), 737-744. https://doi.org/10.1038/nmat4294

Konwar, A., Gogoi, A., \& Chowdhury, D. (2015). Magnetic alginate-Fe ${ }_{3} \mathrm{O}_{4}$ hydrogel fiber capable of ciprofloxacin hydrochloride adsorption/separation in aqueous solution. RSC Advances, 5(99), 81573-81582. https://doi.org/10.1039/C5RA16404D

Kulkarni, R. V., Boppana, R., Krishna Mohan, G., Mutalik, S., \& Kalyane, N. V. (2012). pH-responsive interpenetrating network hydrogel beads of poly(acrylamide)-g-carrageenan and sodium alginate for intestinal targeted drug delivery: Synthesis, in vitro and in vivo evaluation. Journal of Colloid and Interface Science, 367(1), 509-517. https://doi.org/10.1016/j.jcis.2011.10.025

Lai, J.-Y. (2010). Biocompatibility of chemically cross-linked gelatin hydrogels for ophthalmic use. Journal of Materials Science: Materials in Medicine, 21(6), 1899-1911. https://doi.org/10.1007/s10856010-4035-3 
Lee, C., Shin, J., Lee, J. S., Byun, E., Ryu, J. H., Um, S. H., ... Cho, S.-W. (2013). Bioinspired, Calcium-Free Alginate Hydrogels with Tunable Physical and Mechanical Properties and Improved Biocompatibility. Biomacromolecules, 14(6), 2004-2013. https://doi.org/10.1021/bm400352d

Lee, K. Y., \& Mooney, D. J. (2012). Alginate: Properties and biomedical applications. Progress in Polymer Science, 37(1), 106-126. https://doi.org/10.1016/J.PROGPOLYMSCI.2011.06.003

Li, J., \& Mooney, D. J. (2016). Designing hydrogels for controlled drug delivery. Nature Reviews Materials, 1(12), 16071. https://doi.org/10.1038/natrevmats.2016.71

Li, L., Wang, Y., Pan, L., Shi, Y., Cheng, W., Shi, Y., \& Yu, G. (2015). A Nanostructured Conductive Hydrogels-Based Biosensor Platform for Human Metabolite Detection. Nano Letters, 15(2), 11461151. https://doi.org/10.1021/nl504217p

Li, X., Sun, Q., Li, Q., Kawazoe, N., \& Chen, G. (2018). Functional Hydrogels With Tunable Structures and Properties for Tissue Engineering Applications. Frontiers in Chemistry, 6, 499. https://doi.org/10.3389/fchem.2018.00499

Liu, S., \& Guo, W. (2018). Anti-Biofouling and Healable Materials: Preparation, Mechanisms, and Biomedical Applications. Advanced Functional Materials, 28(41), 1800596. https://doi.org/10.1002/adfm.201800596

Long, J., Li, X., Wu, Z., Xu, E., Xu, X., Jin, Z., \& Jiao, A. (2015). Immobilization of pullulanase onto activated magnetic chitosan/Fe3O4 nanoparticles prepared by in situ mineralization and effect of surface functional groups on the stability. Colloids and Surfaces A: Physicochemical and Engineering Aspects, 472, 69-77. https://doi.org/10.1016/J.COLSURFA.2015.02.038

Macosko, C. W. (1994). Rheology: Principles, Measurements, and Applications ABOUT THE AUTHOR. Wiley-VCH. Retrieved from https://www.wiley.com/en-us

Mahinroosta, M., Jomeh Farsangi, Z., Allahverdi, A., \& Shakoori, Z. (2018). Hydrogels as intelligent materials: A brief review of synthesis, properties and applications. Materials Today Chemistry, 8, 42-55. https://doi.org/10.1016/J.MTCHEM.2018.02.004

Mantha, S., Pillai, S., Khayambashi, P., Upadhyay, A., Zhang, Y., Tao, O., ... Tran, S. D. (2019). Smart Hydrogels in Tissue Engineering and Regenerative Medicine. Materials, 12(20), 3323. https://doi.org/10.3390/ma12203323

Marcus, M., Skaat, H., Alon, N., Margel, S., \& Shefi, O. (2015). NGF-conjugated iron oxide nanoparticles promote differentiation and outgrowth of PC12 cells. Nanoscale, 7(3), 1058-1066. https://doi.org/10.1039/C4NR05193A

Melendres, C. A., O'Leary, T. J., \& Solis, J. (1991). Effect of thiocyanate on the corrosion and passivation 
behaviour of copper and iron: laser Raman spectroscopy and photoelectrochemical studies. Electrochimica Acta, 36(3-4), 505-511. https://doi.org/10.1016/0013-4686(91)85134-S

Moghimi, E., Jacob, A. R., Koumakis, N., \& Petekidis, G. (2017). Colloidal gels tuned by oscillatory shear. Soft Matter, 13(12), 2371-2383. https://doi.org/10.1039/c6sm02508k

Morris, E. R., Rees, D. A., \& Thom, D. (1980). Characterisation of alginate composition and blockstructure by circular dichroism. Carbohydrate Research, 81(2), 305-314. https://doi.org/10.1016/S0008-6215(00)85661-X

Osterholtz, F. D., \& Pohl, E. R. (1992). Kinetics of the Hydrolysis and Condensation of Organofunctional Alkoxysilanes: A Review. Journal of Adhesion Science and Technology, 6(1), 127-149. https://doi.org/10.1163/156856192X00106

Park, S., Lee, Y., Kim, D. N., Park, S., Jang, E., \& Koh, W.-G. (2009). Entrapment of enzyme-linked magnetic nanoparticles within poly(ethylene glycol) hydrogel microparticles prepared by photopatterning. Reactive and Functional Polymers, 69(5), 293-299. https://doi.org/10.1016/J.REACTFUNCTPOLYM.2009.02.001

Peppas, N. A., Hilt, J. Z., Khademhosseini, A., \& Langer, R. (2006). Hydrogels in Biology and Medicine: From Molecular Principles to Bionanotechnology. Advanced Materials, 18(11), 1345-1360. https://doi.org/10.1002/adma.200501612

Prencipe, I., Dellasega, D., Zani, A., Rizzo, D., \& Passoni, M. (2015). Energy dispersive x-ray spectroscopy for nanostructured thin film density evaluation. Science and Technology of Advanced Materials, 16(2), 025007. https://doi.org/10.1088/1468-6996/16/2/025007

Qiu, Y., \& Park, K. Environment-sensitive hydrogels for drug delivery. Advanced Drug Delivery Reviews, (3). Retrieved from https://www.sciencedirect.com/science/article/pii/S0169409X01002034?via\%3Dihub

Qureshi, D., Nayak, S. K., Maji, S., Anis, A., Kim, D., \& Pal, K. (2019). Environment sensitive hydrogels for drug delivery applications. European Polymer Journal, 120, 10922. https://doi.org/10.1016/J.EURPOLYMJ.2019.109220

Ravald, L. A., Chilver, J. W., \& Williams, R. (2007). The corrosion of mild steel by aqueous ammonium thiocyanate. Journal of Applied Chemistry, 7(3), 113-117. https://doi.org/10.1002/jctb.5010070304

Seliktar, D. (2012). Designing cell-compatible hydrogels for biomedical applications. Science (New York, N.Y.), 336(6085), 1124-1128. https://doi.org/10.1126/science.1214804

Soon-Shiong, P., Heintz, R. E., Merideth, N., Yao, Q. X., Yao, Z., Zheng, T., ... Sandford, P. A. (1994). Insulin independence in a type 1 diabetic patient after encapsulated islet transplantation. The Lancet, 
343(8903), 950-951. https://doi.org/10.1016/S0140-6736(94)90067-1

784 Supramaniam, J., Adnan, R., Mohd Kaus, N. H., \& Bushra, R. (2018). Magnetic nanocellulose alginate hydrogel beads as potential drug delivery system. International Journal of Biological Macromolecules, 118, 640-648. https://doi.org/10.1016/J.IJBIOMAC.2018.06.043

Zhu, Y., Zheng, Y., Wang, F., \& Wang, A. (2016). Fabrication of magnetic macroporous chitosan-g-poly

Tanasa, E., Zaharia, C., Radu, I.-C., Surdu, V.-A., Vasile, B. S., Damian, C.-M., \& Andronescu, E. (2019). Novel Nanocomposites Based on Functionalized Magnetic Nanoparticles and Polyacrylamide: Preparation and Complex Characterization. Nanomaterials, 9(10), 1384. https://doi.org/10.3390/nano9101384

Tu, Y., Chen, N., Li, C., Liu, H., Zhu, R., Chen, S., ... He, L. (2019). Advances in injectable self-healing biomedical hydrogels. Acta Biomaterialia, 90, 1-20. https://doi.org/10.1016/J.ACTBIO.2019.03.057

Ulijn, R. V., Bibi, N., Jayawarna, V., Thornton, P. D., Todd, S. J., Mart, R. J., ... Gough, J. E. (2007). Bioresponsive hydrogels. Materials Today, 10(4), 40-48. https://doi.org/10.1016/S13697021(07)70049-4

Wang, H., Jiang, L., Wu, H., Zheng, W., Kan, D., Cheng, R., ... Sun, S.-K. (2019). Biocompatible lodineStarch-Alginate Hydrogel for Tumor Photothermal Therapy. ACS Biomaterials Science \& Engineering, 5(7), 3654-3662. https://doi.org/10.1021/acsbiomaterials.9b00280 (acrylic acid) hydrogel for removal of $\mathrm{Cd} 2+$ and $\mathrm{Pb} 2+$. International Journal of Biological Macromolecules, 93, 483-492. https://doi.org/10.1016/J.IJBIOMAC.2016.09.005 